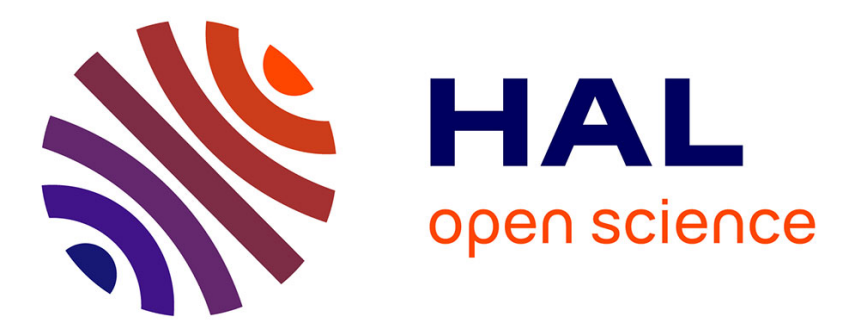

\title{
Error estimates for stochastic differential games: the adverse stopping case
}

\author{
J. Frederic Bonnans, Stefania Maroso, Hasnaa Zidani
}

\section{To cite this version:}

J. Frederic Bonnans, Stefania Maroso, Hasnaa Zidani. Error estimates for stochastic differential games: the adverse stopping case. [Research Report] RR-5441, INRIA. 2004, pp.28. inria-00070566

\section{HAL Id: inria-00070566 \\ https://hal.inria.fr/inria-00070566}

Submitted on 19 May 2006

HAL is a multi-disciplinary open access archive for the deposit and dissemination of scientific research documents, whether they are published or not. The documents may come from teaching and research institutions in France or abroad, or from public or private research centers.
L'archive ouverte pluridisciplinaire HAL, est destinée au dépôt et à la diffusion de documents scientifiques de niveau recherche, publiés ou non, émanant des établissements d'enseignement et de recherche français ou étrangers, des laboratoires publics ou privés. 
INSTITUT NATIONAL DE RECHERCHE EN INFORMATIQUE ET EN AUTOMATIQUE

\title{
Error estimates for stochastic differential games: the adverse stopping case
}

\author{
J. Frédéric BONNANS — Stefania MAROSO — Housnaa ZIDANI
}

\section{$\mathbf{N}^{\circ} \mathbf{5 4 4 1}$}

Décembre 2004

Thème NUM 



\title{
Error estimates for stochastic differential games: the adverse stopping case
}

\author{
J. Frédéric BONNANS* ${ }^{*}$ Stefania MAROSO ${ }^{\dagger}$, Housnaa ZIDANI ${ }^{\ddagger}$ \\ Thème NUM — Systèmes numériques \\ Projet SYDOCO \\ Rapport de recherche $\mathrm{n}^{\circ} 5441$ - Décembre 2004 - 28 pages
}

\begin{abstract}
We obtain error bounds for monotone approximation schemes of a particular Isaacs equation. This is an extension of the theory for estimating errors for the HamiltonJacobi-Bellman equation.

For obtaining upper error bound, we consider the "Krylov regularization" of the Isaacs equation to build an approximate sub-solution of the scheme. To get lower error bound we extend the method of Barles and Jakobsen [2] which consists in introducing a switching system whose solutions are local super-solutions of the Isaacs equation.
\end{abstract}

Key-words: Isaacs equation, Hamilton-Jacobi-Bellman equation, stochastic differential games, finite differences, error estimates.

* J. Frédéric BONNANS: Projet Sydoco, Inria-Rocquencourt, Domaine de Voluceau, BP 105, 78153 Le Chesnay, France (Frederic.Bonnans@inria.fr).

$\dagger$ Stefania MAROSO: Projet Sydoco, Inria-Rocquencourt, Domaine de Voluceau, BP 105, 78153 Le Chesnay, France (Stefania.Maroso@inria.fr).

¥ Housnaa ZIDANI: Projet Sydoco, Inria-Rocquencourt and Unité de Mathématiques Appliquées, ENSTA, 32 Boulevard Victor, 75739 Paris Cedex 15, France (zidani@ensta.fr). 


\section{Estimations d'erreur pour des jeux différentiels stochastiques : le cas de l'arrêt adverse}

Résumé : Nous obtenons des estimations d'erreurs pour des schémas d'approximation monotones d'une équation d'Isaacs particulière. Ceci constitue une extension de la théorie des estimations d'erreurs pour l'équation de Hamilton-Jacobi-Bellman.

Le majorant de l'erreur est obtenue par la méthode de "régularisation de Krylov", qui permet la construction d'une sous-solution approchée du schéma. Le minorant est obtenu en étendant la méthode de Barles and Jakobsen [2], qui consiste en l'introduction d'un système à commutation dont les solutions sont des sursolutions locales de l'équation d'Isaacs.

Mots-clés : Equation d'Isaacs, équation de Hamilton-Jacobi-Bellman, jeux différentiels stochastiques, différences finies, estimations d'erreur. 


\section{Introduction}

The aim of this paper is to give error bounds for approximation schemes of a particular non convex Isaacs equation. More precisely we consider the following equation

$$
\min \left\{\sup _{\alpha \in \mathcal{A}} L^{\alpha}(x, \mathcal{D} u(x)) ; u(x)-\psi(x)\right\}=0, \quad x \in \mathbb{R}^{N},
$$

where

$$
\begin{aligned}
& L^{\alpha}(x, \mathcal{D} u(x))=L^{\alpha}\left(x, u(x), D u(x), D^{2} u(x)\right), \\
& L^{\alpha}(x, t, p, X)=-\operatorname{tr}\left[a^{\alpha}(x) X\right]-b^{\alpha}(x) p+c^{\alpha}(x) t-f^{\alpha}(x) .
\end{aligned}
$$

Here $\mathcal{A}=\left\{\alpha_{1}, \ldots, \alpha_{M}\right\}$ denotes the set of controls, assumed to be finite; the case of a compact set will be dealt with in section 4.3. The coefficients $\left(a^{\alpha}, b^{\alpha}, c^{\alpha}, f^{\alpha}\right)$ are, for each $\alpha \in \mathcal{A}$, bounded and Lipschitz functions $\mathbb{R}^{N} \rightarrow \mathcal{S}^{N} \times \mathbb{R}^{N} \times \mathbb{R} \times \mathbb{R}$, where $\mathcal{S}^{N}$ denotes the set of $N \times N$ symmetric matrices; $\psi$ is a bounded Lipschitz function from $\mathbb{R}^{N}$ into $\mathbb{R}$. Under classical assumptions, (1) has a unique bounded viscosity solution, denoted $u$. The regularity of $u$ depends on the properties of the coefficients $a, b, c, f$.

This problem is a particular case of stochastic differential games, called the adverse stopping case. In fact, we can note that in (1) we have two players, A and B. Player A has a set of controls and wants to minimize the gain. Player B can only decide to stop the game with the objective of maximizing the gain.

Then we consider monotone approximation schemes of (1), of the following form:

$$
\min \left\{S\left(h, x, u_{h}(x), u_{h}\right) ; u_{h}(x)-\psi(x)\right\}=0, \quad x \in \mathbb{R}^{N},
$$

where $S$ is a consistent, monotonic and uniformly continuous approximation of $\sup _{\alpha \in \mathcal{A}} L^{\alpha}$. We will note $u_{h} \in C_{b}\left(\mathbb{R}^{N}\right)$ the solution of $(2)$, which is the approximation of $u$, and $h$ the mesh size. This abstract notations was introduced by Barles and Souganidis [3] to display clearly the monotonicity of the scheme: $S(h, x, r, v)$ is non decreasing in $r$ and non increasing in $v$. Typical approximation schemes that we will consider are Classical Finite Differences [15], Generalized Finite Differences [5] and [4], and Markov Chain Approximations [15].

Until now, results on convergence rates for monotone approximation schemes of the equation with one player have been obtained; i.e. for the following equation:

$$
\sup _{\alpha \in \mathcal{A}} L^{\alpha}(x, \mathcal{D} u(x))=0, \quad x \in \mathbb{R}^{N},
$$

and the scheme

$$
S\left(h, x, u_{h}(x), u_{h}\right)=0, \quad x \in \mathbb{R}^{N} .
$$

Error estimates for this equation have been obtained by Krylov [13],[14] and these results were extended by Barles and Jakobsen [1],[2].

During the redactions of this paper we learned that also Jakobsen was working on the convergence rate for monotone approximations of (1) (see [11]). He obtains error estimates 
in the case of finite differences scheme with matrix $a$ independent of $x$, using a penalization approach.

By using the methods introduced by Barles and Jakobsen [2], we give convergence rate for monotonic approximation schemes of the two player equation. The upper estimate of $u-u_{h}$ is easier to obtain than the lower. The proof involves a "Krylov regularization" of (1), i.e. the perturbed equation

$$
\min \left\{\sup _{\alpha,|e| \leq \varepsilon} L^{\alpha}\left(x+e, \mathcal{D} u^{\varepsilon}(x)\right) ; u^{\varepsilon}(x)-\psi(x)\right\}=0, \quad x \in \mathbb{R}^{N},
$$

and its viscosity solution $u^{\varepsilon}$. A regularization of $u^{\varepsilon}$ by convolution gives an approximate smooth sub-solution of (1), denoted $u_{\varepsilon}$ which is also an approximate sub-solution of (2). So, by using the consistency property, we obtain the upper bound, after choosing an optimal parameter of regularization. Unfortunately we can't proceed in a similar way to build a smooth super-solution of (1) which would lead to the lower estimate on $u-u_{h}$. Instead of a smooth super-solution we build a sequence of local smooth super-solution. In particular we introduce the following switching system which approximates (1)

$$
\min \left\{\max \left\{L^{\alpha_{i}}\left(x, \mathcal{D} v_{i}(x)\right) ; v_{i}(x)-\min _{j \neq i}\left\{v_{j}(x)+k\right\}\right\} ; v_{i}(x)-\psi(x)\right\}=0,
$$

for $x \in \mathbb{R}^{N}$, and $i \in \mathcal{I}=\{1, \ldots, M\}$. For literature on the switching systems, see [6], [8], [9] and [10]. We consider the viscosity solution $v=\left(v_{1}, \ldots, v_{M}\right)$ of this system, and give an estimate of the rate of convergence of $v$ to $u$. Then we consider a perturbed system

$$
\min \left\{\max \left\{\inf _{|e| \leq \varepsilon} L^{\alpha_{i}}\left(x+e, \mathcal{D} v_{i}^{\varepsilon}(x)\right) ; v_{i}^{\varepsilon}(x)-\min _{j \neq i}\left\{v_{j}^{\varepsilon}(x)+k\right\}\right\} ; v_{i}^{\varepsilon}(x)-\psi(x)\right\}=0,
$$

for all $i \in \mathcal{I}$ and $x \in \mathbb{R}^{N}$, and its viscosity solution denoted $v^{\varepsilon}=\left(v_{1}^{\varepsilon}, \ldots, v_{M}^{\varepsilon}\right)$. We regularize $v^{\varepsilon}$ by convolution obtaining $v_{\varepsilon}$, and this function allows to build a local super-solution of (1). Then by applying the consistency and the monotonicity of the scheme we obtain the desired bound. With our result, we can prove an upper bound of $h^{1 / 2}$ and a lower bound of $h^{1 / 5}$ for classical finite differences scheme and for generalized finite differences scheme.

The paper is organized as follows: section 2 introduces the assumptions on equation (1) and scheme (2). Section 3 obtains the rate of convergence of the solution $v$ of (6) to $u$. The first part of section 4 obtains an upper bound of $u-u_{h}$, and the second part of this section uses the rate obtained in section 3 for giving the lower bound of $u-u_{h}$. Section 5 applies our results to the generalized finite difference scheme taken from [5], and studies conditions under those a general Markov chain approximation give better estimates than this scheme. Finally the Appendix gives some auxiliary theorems which are used throughout the paper. We conclude this introduction with some notations. In the sequel $C$ is a positive constant independent on parameters $\varepsilon$ and $h$. By $|\cdot|$ we mean the standard Euclidean norm in any $\mathbb{R}^{M}$ type space. In particular, if $X \in \mathcal{S}^{N}$, then $|X|^{2}=\operatorname{tr}\left(X X^{\top}\right)$, where $X^{\top}$ is the transpose of $X$, i.e. $|X|$ is the Frobenius norm. If $g$ is a bounded function from $\mathbb{R}^{N}$ into either $\mathbb{R}, \mathbb{R}^{M}$, or the space of $N \times P$ matrices, we set

$$
|g|_{0}:=\sup _{x \in \mathbb{R}^{N}}|g(x)| .
$$

INRIA 
If $g$ is also Lipschitz continuous, we set

$$
[g]_{1}:=\sup _{\substack{x, y \in \mathbb{R}^{N} \\ x \neq y}} \frac{|g(x)-g(y)|}{|x-y|}, \quad|g|_{1}:=|g|_{0}+[g]_{1} .
$$

We denote by $\leq$ the component wise ordering in $\mathbb{R}^{N}$, and by $\preceq$ the ordering in the sense of positive semidefinite matrices in $\mathcal{S}(N)$. The space $C_{b}\left(\mathbb{R}^{N}\right)$ (resp. $C_{b, l}\left(\mathbb{R}^{N}\right)$ ) will denote the space of continuous and bounded functions (resp. bounded and Lipschitz functions) from $\mathbb{R}^{N}$ to $\mathbb{R}$.

\section{Well-posedness of the Isaacs equation and of the scheme}

Throughout this paper, we suppose that the following assumptions are satisfied:

(A1) There exist $\lambda, K$ such that, for all $x \in \mathbb{R}^{N}$ and $\alpha \in \mathcal{A}, a^{\alpha}(x)=\frac{1}{2} \sigma^{\alpha}(x)\left(\sigma^{\alpha}(x)\right)^{T}$, where $\sigma^{\alpha}(x)$ is, for each $x$, a $N \times P$ matrix, and

$$
c^{\alpha} \geq \lambda>0 ; \quad\left|\sigma^{\alpha}\right|_{1}+\left|b^{\alpha}\right|_{1}+\left|c^{\alpha}\right|_{1}+\left|f^{\alpha}\right|_{1} \leq K,
$$

(A2) $\min \{1, \lambda\}>\sup _{\alpha}\left\{\left[\sigma^{\alpha}\right]_{1}+\left[b^{\alpha}\right]_{1}\right\}$.

Definition 1 The function $u \in C\left(\mathbb{R}^{N}\right)$ is called a viscosity sub-solution (resp. supersolution) of (1) if, for every $x \in \mathbb{R}^{N}$,

$$
\min \left\{\sup _{\alpha} L^{\alpha}\left(x, u(x), D \varphi(x), D^{2} \varphi(x)\right) ; u(x)-\psi(x)\right\} \leq 0, \quad(\text { resp. } \quad \geq 0),
$$

for each $\varphi \in C^{2}\left(\mathbb{R}^{N}\right)$ such that $u-\varphi$ has a local maximum (resp. a local minimum) at $x$. Finally we call $u$ a viscosity solution of (1) if it is both a sub-solution and a super-solution.

We refer to [11, lemma A.1] for the proof of the following result.

Proposition 2 Assume (A1) and (A2). Then the following statements hold:

(i) If $u_{1}$ and $u_{2}$ are respectively viscosity sub-solution and viscosity super-solution of (1), $u_{1}, u_{2} \in C_{b}\left(\mathbb{R}^{N}\right)$, then $u_{1} \leq u_{2}$ in $\mathbb{R}^{N}$.

(ii) There exists an unique viscosity solution $u$ of (1), in the space $C_{b, l}\left(\mathbb{R}^{N}\right)$.

Consider the scheme (2), with $h>0$ and $S: \mathbb{R}^{+} \times \mathbb{R}^{N} \times \mathbb{R} \times C_{b}\left(\mathbb{R}^{N}\right) \rightarrow \mathbb{R}$. We make the following assumptions:

(S1) Monotonicity: for all $h>0, r \in \mathbb{R}^{N}, m \geq 0, x \in \mathbb{R}^{N}$ and bounded and continuous functions $u, v$ such that $u \leq v$ in $\mathbb{R}^{N}$,

$$
S(h, x, r+m, u+m) \geq \lambda m+S(h, x, r, v) .
$$

RR $n^{\circ} 5441$ 
(S2) Regularity: for all $h>0$ and $\phi \in C_{b}\left(\mathbb{R}^{N}\right), x \mapsto S(h, x, \phi(x), \phi)$ is bounded and continuous; $r \mapsto S(h, x, r, \phi)$ is uniformly continuous for bounded $r$, uniformly with respect to $x \in \mathbb{R}^{N}$.

(S3) There exist $n, k_{i}>0, i \in J \subseteq\{1, \ldots, n\}$ and a constant $K_{c}>0$ such that for all $h>0$ and $x$ in $\mathbb{R}^{N}$, and for every smooth $\phi \in C^{n}\left(\mathbb{R}^{N}\right)$ such that $\left|D^{i} \phi\right|_{0}$ is bounded, for every $i \in J$, the following holds:

$$
\left|\sup _{\alpha} L^{\alpha}(x, \mathcal{D} \phi)-S(h, x, \phi(x), \phi)\right| \leq K_{c} Q(\phi),
$$

where $Q(\phi):=\sum_{i \in J}\left|D^{i} \phi\right|_{0} h^{k_{i}}$.

Remark 3 (S1) and (S2) imply that $S$ is decreasing w.r.t. $v$ (take $m=0$ ), and increasing w.r.t. $r($ take $v=u+m)$.

In the following, we say that a function $v \in C_{b}\left(\mathbb{R}^{N}\right)$ is a sub-solution (resp. supersolution) to the scheme (2) if

$$
\min \{S(h, x, v(x), v) ; v(x)-\psi(x)\} \leq 0, \quad(\text { resp. } \quad \geq 0), \quad \text { for all } x \in \mathbb{R}^{N} .
$$

Under assumptions (S1) and (S2), we have the existence of a comparison principle for bounded continuous solutions of (2); i.e.

Theorem 4 Let $u_{h}$ (resp. $v_{h}$ ) be a bounded, continuous sub-solution (resp. super-solution) of (2). Then we have $u_{h}(x) \leq v_{h}(x)$, for all $x \in \mathbb{R}^{N}$.

Proof. The proof is an easy extension of [1, lemma 2.3]. We assume that $m:=\sup _{x}\left(u_{h}(x)-\right.$ $\left.v_{h}(x)\right)>0$ and obtain a contradiction. Let $\left\{x_{n}\right\}_{n}$ be a sequence in $\mathbb{R}^{N}$ such that $\delta_{n}:=$ $u_{h}\left(x_{n}\right)-v_{h}\left(x_{n}\right)$ converges to $m$ as $n \rightarrow \infty$. Then $\delta_{n}>0$ for large enough $n$. By using the notion of sub and super-solution, and the fact that $\min (A, B)-\min (C, D) \geq \min (A-$ $C, B-D)$, get

$$
0 \geq \min \left\{S\left(h, x_{n}, u_{h}\left(x_{n}\right), u_{h}\right)-S\left(h, x_{n}, v_{h}\left(x_{n}\right), v_{h}\right) ; u_{h}\left(x_{n}\right)-v_{h}\left(x_{n}\right)\right\} .
$$

Since $u_{h}\left(x_{n}\right)-v_{h}\left(x_{n}\right)=\delta_{n}>0$, by using (S1), we have

$$
\begin{aligned}
0 & \geq S\left(h, x_{n}, u_{h}\left(x_{n}\right), u_{h}\right)-S\left(h, x_{n}, v_{h}\left(x_{n}\right), v_{h}\right) \\
& \geq S\left(h, x_{n}, v_{h}\left(x_{n}\right)+\delta_{n}, v_{h}+m\right)-S\left(h, x_{n}, v_{h}\left(x_{n}\right), v_{h}\right) \\
& \geq S\left(h, x_{n}, v_{h}\left(x_{n}\right)+m, v_{h}+m\right)+\omega\left(m-\delta_{n}\right)-S\left(h, x_{n}, v_{h}\left(x_{n}\right), v_{h}\right) \\
& \geq \lambda m+\omega\left(m-\delta_{n}\right),
\end{aligned}
$$

where $\omega(t) \rightarrow 0$ when $t \rightarrow 0^{+}$is given by (S2). Letting $n \rightarrow \infty$ yields $m \leq 0$ which is a contradiction. 
In all the sequel we will use a sequence of mollifiers $\left(\rho_{\varepsilon}\right)_{\varepsilon}$ defined as follows:

$$
\rho_{\varepsilon}(x)=\varepsilon^{-N} \rho(x / \varepsilon),
$$

where $\rho \in C^{\infty}\left(\mathbb{R}^{N}\right), \int_{\mathbb{R}^{N}} \rho=1, \operatorname{supp}\{\rho\} \subseteq \bar{B}(0,1)$ and $\rho \geq 0$. If $g$ is a continuous function of $\mathbb{R}^{N}$ to $\mathbb{R}$, then we define the mollification of $g$ as follows:

$$
g_{\varepsilon}(x):=\int_{\mathbb{R}^{N}} g(x-e) \rho_{\varepsilon}(e) d e .
$$

Moreover, if $g$ is a Lipschitz function, then

$$
\left|g(x)-g_{\varepsilon}(x)\right| \leq \varepsilon[g]_{1} .
$$

If $g \in C_{b}^{n}\left(\mathbb{R}^{N}\right)\left(\operatorname{resp} . C_{b, l}^{n}\left(\mathbb{R}^{N}\right)\right)$, then

$$
\left|D^{i} g_{\varepsilon}(x)\right| \leq C \varepsilon^{-i}|g|_{0}, \quad\left(\text { resp. } C \varepsilon^{1-i}|g|_{0}\right), \quad \forall i=1, \ldots, n .
$$

\section{Switching system}

Consider the following switching system approximation of (1):

$$
\min \left\{\max \left(L^{\alpha_{i}}\left(x, \mathcal{D} v_{i}(x)\right) ; v_{i}(x)-\min _{j \neq i}\left\{v_{j}(x)+k\right\}\right) ; v_{i}(x)-\psi(x)\right\}=0,
$$

for $i \in \mathcal{I}=\{1, \ldots, M\}$ and $x \in \mathbb{R}^{N}$. In particular we have an equation for every control. A viscosity solution theory for the switching system can be found in [9], [10], [16]. We recall here the definition of viscosity solution for a general switching system of the form:

$$
F_{i}\left(x, v, D v_{i}, D^{2} v_{i}\right)=0, \quad i=1, \ldots, M,
$$

where $F_{i}: \mathbb{R}^{N} \times \mathbb{R}^{M} \times \mathbb{R}^{N} \times \mathcal{S}^{N} \rightarrow \mathbb{R}$.

Definition 5 The function $v=\left(v_{1}, \ldots, v_{M}\right) \in C\left(\mathbb{R}^{N}\right)^{M}$ is called a viscosity sub-solution (resp. super-solution) of (13) if, for every $i \in \mathcal{I}$ and $x \in \mathbb{R}^{N}$,

$$
F_{i}\left(x, v(x), D \varphi(x), D^{2} \varphi(x)\right) \leq 0, \quad(\text { resp. } \geq 0),
$$

for each $\varphi \in C^{2}\left(\mathbb{R}^{N}\right)$ such that $v_{i}-\varphi$ has a local maximum (resp. a local minimum) at $x$. Finally we call $v$ a viscosity solution of (13) if it is both a sub-solution and a super-solution.

Lemma 20 implies a comparison principle for (12), and then the existence of a unique viscosity solution of $(12)$ in $C_{b, l}\left(\mathbb{R}^{N}\right)^{M}$, denoted $v=\left(v_{1}, \ldots, v_{M}\right)$.

We perturb the system (12) and build the following auxiliary system

$$
\min \left\{\max \left(\sup _{|e| \leq \varepsilon} L^{\alpha_{i}}\left(x+e, \mathcal{D} v_{i}^{\varepsilon}(x)\right) ; v_{i}^{\varepsilon}(x)-\min _{j \neq i}\left\{v_{j}^{\varepsilon}(x)+k\right\}\right) ; v_{i}^{\varepsilon}(x)-\psi(x)\right\}=0 .
$$

Lemma 20 applied to (14), implies the existence of a unique viscosity solution of (14), denoted $v^{\varepsilon}=\left(v_{1}^{\varepsilon}, \ldots, v_{M}^{\varepsilon}\right)$, in $C_{b, l}\left(\mathbb{R}^{N}\right)^{M}$. The following lemma is consequence of theorem 21.

$\mathrm{RR} \mathrm{n}^{\circ} 5441$ 
Lemma 6 Under assumptions (A1) and (A2), we have that

$$
\left|v_{i}-v_{i}^{\varepsilon}\right| \leq C \varepsilon,
$$

where $C$ only depends on $K, \lambda$ and $[\psi]_{1}$.

For every $i$, let $v_{i \varepsilon}$ the mollification of $v_{i}^{\varepsilon}$, defined as in (9). Since $v_{i}^{\varepsilon}$ is a Lipschitz function, uniformly w.r.t $\varepsilon>0$ sufficiently small, (10) implies

$$
\left|v_{i}^{\varepsilon}(x)-v_{i \varepsilon}(x)\right| \leq \max _{i}\left[v_{i}^{\varepsilon}\right]_{1} \varepsilon
$$

lemma 20 implies that $\max _{i}\left[v_{i}^{\varepsilon}\right]_{1}$ remains bounded when $\varepsilon \downarrow 0$ (this argument will be used several times in the paper).

Lemma 7 The function $v_{i \varepsilon}-R$ is, for all $i$, sub-solution of equation (1), for some

$$
R:=C\left(k+\varepsilon+\frac{k}{\varepsilon^{2}}\right),
$$

where the constant $C$ depends only on $K, \lambda$ and $[\psi]_{1}$.

Proof. Let $R$ satisfy (17). We have to prove that, for large enough $C$,

$$
\min \left\{\sup _{\alpha} L^{\alpha}\left(x, \mathcal{D}\left(v_{i \varepsilon}(x)-R\right) ; v_{i \varepsilon}(x)-R-\psi(x)\right\} \leq 0, \quad \forall x \in \mathbb{R}^{N},\right.
$$

for all $i \in \mathcal{I}$. Since $v_{i \varepsilon} \in C^{\infty}\left(\mathbb{R}^{N}\right)$, the definition of viscosity sub-solution is equivalent to the notion of classical sub-solution. Therefore we have to prove that one of the following statements holds for all $x \in \mathbb{R}^{N}$ :

$$
\begin{aligned}
& v_{i \varepsilon}(x)-R \leq \psi(x), \quad \forall i \in \mathcal{I}, \\
& \sup _{\alpha} L^{\alpha}\left(x, \mathcal{D}\left(v_{i \varepsilon}(x)-R\right)\right) \leq 0, \quad \forall i \in \mathcal{I} .
\end{aligned}
$$

For every $x \in \mathbb{R}^{N}$, set

$$
I^{\varepsilon}(x):=\left\{i \in \mathcal{I} \mid v_{i}^{\varepsilon}(x)=\psi(x)\right\} .
$$

Let $\tilde{x} \in \mathbb{R}^{N}$. Denote by $B(\tilde{x}, 2 \varepsilon)$ the ball centered on $\tilde{x}$ with radius $2 \varepsilon$. Then we have the two following possibilities:

CASE A: There exists $y \in B(\tilde{x}, 2 \varepsilon)$ such that $I^{\varepsilon}(y) \neq \varnothing$. We claim that (19a) holds. We have $v_{i_{0}}^{\varepsilon}(y)=\psi(y)$, for some $i_{0} \in I^{\varepsilon}(y)$. Let $i \notin I^{\varepsilon}(y)$. The function $v_{i}^{\varepsilon}(x)-|x-y|^{2} / \varepsilon_{1}$ has, for sufficiently small $\varepsilon_{1}>0$, a local maximum at a point $x_{\varepsilon}$ such that $\left|x_{\varepsilon}-y\right| \leq \varepsilon$. Since $v^{\varepsilon}$ is the viscosity solution of (14), one of the following statements holds:

$$
\begin{aligned}
& v_{i}^{\varepsilon}\left(x_{\varepsilon}\right) \leq \psi\left(x_{\varepsilon}\right), \\
& \max \left\{\sup _{|e| \leq \varepsilon} L^{\alpha_{i}}\left(x_{\varepsilon}+e, v_{i}^{\varepsilon}\left(x_{\varepsilon}\right), \frac{2}{\varepsilon_{1}}\left(x_{\varepsilon}-y\right), \frac{2 I}{\varepsilon_{1}}\right) ;\right. \\
&\left.v_{i}^{\varepsilon}\left(x_{\varepsilon}\right)-\min _{j \neq i}\left\{v_{j}^{\varepsilon}\left(x_{\varepsilon}\right)+k\right\}\right\} \leq 0 .
\end{aligned}
$$

INRIA 
If $v_{i}^{\varepsilon}\left(x_{\varepsilon}\right) \leq \psi\left(x_{\varepsilon}\right)$, since $v_{i}^{\varepsilon}$ and $\psi$ are Lipschitz, we obtain

$$
v_{i}^{\varepsilon}(y) \leq \psi(y)+\left([\psi]_{1}+\max _{i}\left[v_{i}^{\varepsilon}\right]_{1}\right) \varepsilon
$$

Otherwise, with (21b), we have

$$
v_{i}^{\varepsilon}(y) \leq \max _{i}\left[v_{i}^{\varepsilon}\right]_{1} \varepsilon+v_{i_{0}}^{\varepsilon}\left(x_{\varepsilon}\right)+k \leq 2 \max _{i}\left[v_{i}^{\varepsilon}\right]_{1} \varepsilon+v_{i_{0}}^{\varepsilon}(y)+k .
$$

Since either (22) or (23) holds, we deduce that

$$
v_{i}^{\varepsilon}(y)-C \varepsilon-k \leq \psi(y), \quad \forall i \in \mathcal{I},
$$

where $C$ depends on $[\psi]_{1}$ and $\max _{i}\left[v_{i}^{\varepsilon}\right]_{1}$. Since $y \in B(\tilde{x}, 2 \varepsilon)$, and $\psi$ and $v_{i}^{\varepsilon}$ are Lipschitz, this implies $v_{i}^{\varepsilon}(\tilde{x}) \leq \psi(\tilde{x})+k+C \varepsilon$, for all $i \in \mathcal{I}$. Applying (16), obtain $v_{i \varepsilon}(\tilde{x}) \leq \psi(\tilde{x})+R$, for all $i \in \mathcal{I}$, which implies (18).

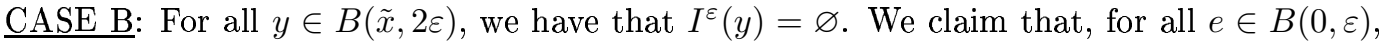
$\left(v_{1}^{\varepsilon}(\cdot-e), \ldots, v_{M}^{\varepsilon}(\cdot-e)\right)$ is a viscosity sub-solution of the following system

$$
\max \left\{L^{\alpha_{i}}\left(x, \mathcal{D} w_{i}(x)\right) ; w_{i}(x)-\min _{j \neq i}\left\{w_{j}(x)+k\right\}\right\}=0, \quad x \in B(\tilde{x}, \varepsilon) .
$$

Fix $e_{1} \in B(0, \varepsilon)$ and $i \in \mathcal{I}$. Let $\varphi \in C^{2}\left(\mathbb{R}^{N}\right)$ be such that $v_{i}^{\varepsilon}\left(\cdot-e_{1}\right)-\varphi(\cdot)$ has a local maximum $x_{e_{1}}$ in the ball $B(\tilde{x}, \varepsilon)$. Then $v_{i}^{\varepsilon}(\cdot)-\varphi\left(\cdot+e_{1}\right)$ has a local maximum at $x_{e_{1}}-e_{1}$. Since $x_{e_{1}}-e_{1} \in B(\tilde{x}, 2 \varepsilon)$, we have that $v_{i}^{\varepsilon}\left(x_{e_{1}}-e_{1}\right)>\psi\left(x_{e_{1}}-e_{1}\right)$, and since $v^{\varepsilon}$ is the viscosity solution of $(14)$, we obtain

$$
\begin{gathered}
\max \left\{\sup _{|e| \leq \varepsilon} L^{\alpha_{i}}\left(x_{e_{1}}-e_{1}+e, v_{i}^{\varepsilon}\left(x_{e_{1}}-e_{1}\right), D \varphi\left(x_{e_{1}}\right), D^{2} \varphi\left(x_{e_{1}}\right)\right) ;\right. \\
\left.v_{i}^{\varepsilon}\left(x_{e_{1}}-e_{1}\right)-\min _{j \neq i}\left\{v_{j}^{\varepsilon}\left(x_{e_{1}}-e_{1}\right)+k\right\}\right\} \leq 0 .
\end{gathered}
$$

Taking $e=e_{1}$, obtain

$$
\left\{\begin{array}{l}
L^{\alpha_{i}}\left(x_{e_{1}}, v_{i}^{\varepsilon}\left(x_{e_{1}}-e_{1}\right), D \varphi\left(x_{e_{1}}\right), D^{2} \varphi\left(x_{e_{1}}\right)\right) \leq 0 \\
v_{i}^{\varepsilon}\left(x_{e_{1}}-e_{1}\right)-\min _{j \neq i}\left\{v_{j}^{\varepsilon}\left(x_{e_{1}}-e_{1}\right)+k\right\} \leq 0
\end{array}\right.
$$

This being true for an arbitrary $e_{1} \in B(0, \varepsilon)$ and $i \in \mathcal{I}$, we obtain that, for all $|e| \leq \varepsilon$, $\left(v_{1}^{\varepsilon}(\cdot-e), \ldots, v_{M}^{\varepsilon}(\cdot-e)\right)$ is a viscosity sub-solution of (25). Applying [1, lemma A.3 and lemma 2.7], since $v_{i \varepsilon}(\cdot)$ is limit of convex combination of $v_{i}^{\varepsilon}(\cdot-e)$, for $e \in B(0, \varepsilon)$, then $\left(v_{1 \varepsilon}(\cdot), \ldots, v_{M \varepsilon}(\cdot)\right)$ is a viscosity sub-solution of $(25)$. Moreover, since it is a smooth function, it is a sub-solution of (25) in the classical sense, and we have

$$
L^{\alpha_{i}}\left(\tilde{x}, \mathcal{D} v_{i \varepsilon}(\tilde{x})\right) \leq 0, \quad \forall i \in \mathcal{I} .
$$


We know that $\left|v_{i}^{\varepsilon}(y)-v_{j}^{\varepsilon}(y)\right| \leq k$ for all $i, j \in \mathcal{I}$ and $y \in B(\tilde{x}, \varepsilon)$. Consequently

$$
D^{n} v_{i \varepsilon}(\tilde{x})-D^{n} v_{j \varepsilon}(\tilde{x}) \leq \frac{C k}{\varepsilon^{n}}, \quad \forall n \geq 1,
$$

where $C$ depends only on $\rho$ defined in (8). It follows that

$$
L^{\alpha_{i}}\left(\tilde{x}, \mathcal{D} v_{j \varepsilon}(\tilde{x})\right)-L^{\alpha_{i}}\left(\tilde{x}, \mathcal{D} v_{i \varepsilon}(\tilde{x})\right) \leq \frac{C k}{\varepsilon^{2}}, \quad \forall i, j \in \mathcal{I} .
$$

Combining with (26), get $L^{\alpha_{i}}\left(\tilde{x}, \mathcal{D} v_{j \varepsilon}(\tilde{x})\right) \leq C k / \varepsilon^{2}$, for all $i$ and $j$ in $\mathcal{I}$, for some $C$ depending on $\rho$ and $K$, and hence, $\sup _{\alpha} L^{\alpha}\left(\tilde{x}, \mathcal{D} v_{i \varepsilon}(\tilde{x})\right) \leq C k / \varepsilon^{2}$ for all $i$ in $\mathcal{I}$. Since $L^{\alpha}$ is uniformly Lipschitz, we have that for all $i$ in $\mathcal{I}, \sup _{\alpha} L^{\alpha}\left(\tilde{x}, \mathcal{D} v_{i \varepsilon}(\tilde{x})-C k /\left(\lambda \varepsilon^{2}\right)\right) \leq 0$. Therefore (18) also holds in this case.

Theorem 8 For every $i \in \mathcal{I}$ and for all $x \in \mathbb{R}^{N}$ we have

$$
0 \leq v_{i}-u \leq C k^{1 / 3}
$$

where $C$ depends only on $\lambda$ and $K$ from (A1).

Proof. a) We prove the first inequality of $(27)$. Let $w=(u, \ldots, u)$ be the vector whose $M$ components are equal to $u$. We claim that $w$ is a viscosity sub-solution of (12). Let $\varphi \in C^{2}\left(\mathbb{R}^{N}\right)$ be such that $u-\varphi$ has a local maximum at $x_{0} \in \mathbb{R}^{N}$. Since $u$ is a viscosity sub-solution of (1), either $u\left(x_{0}\right) \leq \psi\left(x_{0}\right)$, or $\sup _{\alpha} L^{\alpha}\left(x_{0}, u\left(x_{0}\right), D \varphi\left(x_{0}\right), D^{2} \varphi\left(x_{0}\right)\right) \leq 0$. If the latter holds, then

$$
L^{\alpha_{i}}\left(x_{0}, u\left(x_{0}\right), D \varphi\left(x_{0}\right), D^{2} \varphi\left(x_{0}\right)\right) \leq 0, \quad \forall i \in \mathcal{I} .
$$

Combining both cases, obtain

$$
\begin{gathered}
\min \left\{\max \left(L^{\alpha_{i}}\left(x_{0}, u\left(x_{0}\right), D \varphi\left(x_{0}\right), D^{2} \varphi\left(x_{0}\right)\right) ; u\left(x_{0}\right)-\min _{j \neq i}\left\{u\left(x_{0}\right)+k\right\}\right) ;\right. \\
\left.u\left(x_{0}\right)-\psi\left(x_{0}\right)\right\} \leq 0, \quad \forall i \in \mathcal{I} .
\end{gathered}
$$

Therefore $w$ is a viscosity sub-solution of (12). By the comparison principle (lemma 20), the first inequality of (27) holds.

b) We now prove the second inequality in (27). By lemma 7 and proposition 2, we have that $v_{i \varepsilon}-R \leq u$, for all $i \in \mathcal{I}$, and $x \in \mathbb{R}^{N}$, where $R$ satisfies (17). Applying (16) and (15), obtain

$$
v_{i}-u \leq\left|v_{i}-v_{i}^{\varepsilon}\right|+\left|v_{i}^{\varepsilon}-v_{i \varepsilon}\right|+\left|v_{i \varepsilon}-u\right| \leq C\left(\frac{k}{\varepsilon^{2}}+\varepsilon+k\right), \quad \forall i \in \mathcal{I}, \quad \forall x \in \mathbb{R}^{N},
$$

where $C$ depends on $K, \lambda, \max _{i}\left[v_{i}^{\varepsilon}\right]_{1},[\psi]_{1}$. Minimizing with respect to $\varepsilon$, we obtain the desired upper bound, with $\varepsilon=k^{1 / 3}$. 
Remark 9 We obtain the same estimate that in the case of only one player, (see [2, theorem 2.3]), by extending the same methods.

\section{Bounds on $u-u_{h}$}

We state in this section our main results: the upper and lower bounds on $u-u_{h}$.

\subsection{Upper bound on $u-u_{h}$}

Perturb the equation (1) so as to obtain

$$
\min \left\{\sup _{\alpha,|e| \leq \varepsilon} L^{\alpha}\left(x+e, \mathcal{D} u^{\varepsilon}(x)\right) ; u^{\varepsilon}(x)-\psi(x)\right\}=0, \quad x \in \mathbb{R}^{N} .
$$

Under assumptions (A1) and(A2), by proposition 2, (28) has a unique viscosity solution $u^{\varepsilon} \in C_{b, l}\left(\mathbb{R}^{N}\right)$. In view of Theorem 21 with $k=0$, we have that $\left|u-u^{\varepsilon}\right| \leq C \varepsilon$, for some $C$ depending on $\lambda, K$ and $[\psi]_{1}$. Define the contact domain of $u^{\varepsilon}$ as

$$
X\left(u^{\varepsilon}\right):=\left\{x \in \mathbb{R}^{N} \mid u^{\varepsilon}(x)=\psi(x)\right\} .
$$

Let $u_{\varepsilon}$ be the mollification of $u^{\varepsilon}$, defined as in (9). Since $u^{\varepsilon}$ is a Lipschitz function, uniformly w.r.t $\varepsilon>0$ sufficiently small, (10) implies

$$
\left|u^{\varepsilon}(x)-u_{\varepsilon}(x)\right| \leq\left[u^{\varepsilon}\right]_{1} \varepsilon,
$$

where $\left[u^{\varepsilon}\right]_{1}$ remains bounded.

Theorem 10 Assume that (A1), (A2), (S1)-(S3) hold and let the approximation scheme (2) have a unique solution $u_{h}$ in $C_{b}\left(\mathbb{R}^{N}\right)$. Then, for sufficiently small $h>0$, we have

$$
u-u_{h} \leq C h^{\ell}, \quad \forall x \in \mathbb{R}^{N},
$$

where $\ell:=\min _{i \in J}\left\{k_{i} / i\right\}, C$ depends only on $\lambda, K,[\psi]_{1}$ and $K_{c}$, the constants $k_{i}$ and $K_{C}$ being defined in (S3).

Proof. We claim that

$$
\min \left\{S\left(h, x, u_{\varepsilon}(x)-R_{1}, u_{\varepsilon}-R_{1}\right) ; u_{\varepsilon}(x)-R_{1}-\psi(x)\right\} \leq 0, \quad \forall x \in \mathbb{R}^{N},
$$

for some $R_{1}>0$ of the form $R_{1}:=\lambda^{-1} Q\left(u_{\varepsilon}\right)+C \varepsilon$, where $Q(\cdot)$ was defined in (S3) and $C$ depends only on $[\psi]_{1}$ and $\left[u^{\varepsilon}\right]_{1}$. Indeed, we will prove a slightly stronger result: for any $x \in \mathbb{R}^{N}$, one at least of the following two statements holds:

$$
\begin{aligned}
& u_{\varepsilon}(x)-C \varepsilon \leq \psi(x), \\
& S\left(h, x, u_{\varepsilon}(x)-K_{C} \lambda^{-1} Q\left(u_{\varepsilon}\right), u_{\varepsilon}-K_{C} \lambda^{-1} Q\left(u_{\varepsilon}\right)\right) \leq 0 .
\end{aligned}
$$


Fix an $\tilde{x} \in \mathbb{R}^{N}$. We have the following alternative:

CASE A: There exists $y \in B(\tilde{x}, 2 \varepsilon)$ such that $y \in X\left(u^{\varepsilon}\right)$, i.e. $u^{\varepsilon}(y)=\psi(y)$. Since $u^{\varepsilon}$ and $\psi$ are uniformly Lipschitz, for some $C$ depending only on $[\psi]_{1}$ and $\left[u^{\varepsilon}\right]_{1}$, we obtain (32a) at point $x=\tilde{x}$.

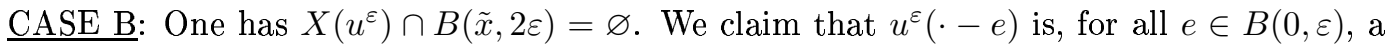
viscosity sub-solution of

$$
\sup _{\alpha} L^{\alpha}(x, \mathcal{D} w(x))=0, \quad x \in B(\tilde{x}, \varepsilon) .
$$

Fix $e_{1} \in B(\tilde{x}, \varepsilon)$, and let $\varphi \in C^{2}\left(\mathbb{R}^{N}\right)$ be such that $u^{\varepsilon}\left(\cdot-e_{1}\right)-\varphi(\cdot)$ has a local maximum at a point $x_{e_{1}} \in B(\tilde{x}, \varepsilon)$. Then $u^{\varepsilon}(\cdot)-\varphi\left(\cdot+e_{1}\right)$ has a local maximum at $x_{e_{1}}-e_{1}$. Since $x_{e_{1}}-e_{1} \in B(\tilde{x}, 2 \varepsilon)$, and hence, $x_{e_{1}}-e_{1} \notin X\left(u^{\varepsilon}\right)$, we have, whenever $|e| \leq \varepsilon$,

$$
\sup _{\alpha,|e| \leq \varepsilon} L^{\alpha}\left(x_{e_{1}}-e_{1}+e, u^{\varepsilon}\left(x_{e_{1}}-e_{1}\right), D \varphi\left(x_{e_{1}}\right), D^{2} \varphi\left(x_{e_{1}}\right)\right) \leq 0 .
$$

Taking $e=e_{1}$, we have

$$
\sup _{\alpha} L^{\alpha}\left(x_{e_{1}}, u^{\varepsilon}\left(x_{e_{1}}-e_{1}\right), D \varphi\left(x_{e_{1}}\right), D^{2} \varphi\left(x_{e_{1}}\right)\right) \leq 0 .
$$

This proves our claim that $u^{\varepsilon}\left(\cdot-e_{1}\right)$ is a viscosity sub-solution of (33). Since $e_{1}$ is an arbitrary point of $B(0, \varepsilon), u^{\varepsilon}(\cdot-e)$ is a viscosity sub-solution of $(33)$, for all $|e| \leq \varepsilon$. Since $u_{\varepsilon}(\cdot)$ is a $C^{\infty}$ function, and it is limit of convex combination of $u^{\varepsilon}(\cdot-e)$ (see [1, lemma A.3 and lemma 2.7]), hence, applying ([1, lemma 2.7]), we can say that $u_{\varepsilon}(\cdot)$ is a sub-solution of (33) in the classical sense. This implies

$$
\sup _{\alpha} L^{\alpha}\left(\tilde{x}, \mathcal{D} u_{\varepsilon}(\tilde{x})\right) \leq 0 .
$$

By consistency, $S\left(h, \tilde{x}, u_{\varepsilon}(\tilde{x}), u_{\varepsilon}\right) \leq K_{C} Q\left(u_{\varepsilon}\right)$. Applying (S1) with $u=v=u_{\varepsilon}-K_{C} \lambda^{-1} Q\left(u_{\varepsilon}\right)$, $r=u_{\varepsilon}(\tilde{x})-K_{C} \lambda^{-1} Q\left(u_{\varepsilon}\right)$, and $m=K_{C} \lambda^{-1} Q\left(u_{\varepsilon}\right)$, obtain $(32 \mathrm{~b})$ at point $x=\tilde{x}$. Combining cases $\mathrm{A}$ and $\mathrm{B}$ we obtain (31). So $u_{\varepsilon}-R_{1}$ is a sub-solution of (2). By theorem 4 , $u_{\varepsilon}-R_{1} \leq u_{h}$, i.e., $u-u_{h} \leq K_{C} \lambda^{-1} Q\left(u_{\varepsilon}\right)+C \varepsilon$, where $C$ depends on $\left[u^{\varepsilon}\right]_{1}$ and $[\psi]_{1}$. Using $Q\left(u_{\varepsilon}\right)=\sum_{i \in J}\left|D^{i} u_{\varepsilon}\right| h^{k_{i}}$, and (11), obtain $Q\left(u_{\varepsilon}\right) \leq C \sum_{i \in J} \varepsilon^{1-i} h^{k_{i}}$. The result follows by setting $\varepsilon=\max _{i \in J} h^{k_{i} / i}$.

\subsection{Lower bound on $u-u_{h}$}

We perturb the switching system (12) as follows

$$
\min \left\{\max \left(\inf _{|e| \leq \varepsilon} L^{\alpha_{i}}\left(x+e, \mathcal{D} v_{i}^{\varepsilon}(x)\right) ; v_{i}^{\varepsilon}(x)-\min _{j \neq i}\left\{v_{j}^{\varepsilon}(x)+k\right\}\right) ; v_{i}^{\varepsilon}(x)-\psi(x)\right\}=0,
$$

INRIA 
for all $i \in \mathcal{I}$ and $x \in \mathbb{R}^{N}$. By lemma 20, this system has a unique viscosity solution $v^{\varepsilon}=\left(v_{1}^{\varepsilon}, \ldots, v_{M}^{\varepsilon}\right)$ in $C_{b, l}\left(\mathbb{R}^{N}\right)^{M}$. Consider $v_{\varepsilon}$, the mollification of $v^{\varepsilon}$, defined as in (9). Since $v^{\varepsilon}$ is a Lipschitz function, uniformly w.r.t. $\varepsilon>0$ sufficiently small, applying lemma (21) and (10) we have

$$
\left|v_{i}-v_{i}^{\varepsilon}\right| \leq C \varepsilon, \quad\left|v_{i}^{\varepsilon}-v_{i \varepsilon}\right| \leq \max _{i}\left[v_{i}^{\varepsilon}\right]_{1} \varepsilon
$$

where $C$ depends on $\lambda, K$ and $[\psi]_{1}$, and $\max _{i}\left[v_{i}^{\varepsilon}\right]_{1}$ remains bounded.

Lemma 11 Let $x_{0} \in \mathbb{R}^{N}, i_{0} \in \arg \min _{j \in \mathcal{I}} v_{j \varepsilon}\left(x_{0}\right)$, and assume that

$$
\varepsilon \leq\left(12 \sup _{i}\left[v_{i}^{\varepsilon}\right]_{1}\right)^{-1} k .
$$

Then the following statements hold

$$
\begin{gathered}
v_{i_{0}}^{\varepsilon}(y)<v_{j}^{\varepsilon}(y)+k, \quad \text { for all } j \in \mathcal{I}, \text { and } y \in B\left(x_{0}, 2 \varepsilon\right), \\
\sup _{\alpha} L^{\alpha}\left(x_{0}, \mathcal{D} v_{i_{0} \varepsilon}\left(x_{0}\right)\right) \geq 0 .
\end{gathered}
$$

Proof. We follow the method of ([2, Lemma 3.4]).

a) Let us prove (38). Since $i_{0} \in \arg \min _{j \in \mathcal{I}} v_{j \varepsilon}\left(x_{0}\right)$,

$$
v_{i_{0} \varepsilon}\left(x_{0}\right)-\min _{j \neq i_{0}}\left\{v_{j \varepsilon}\left(x_{0}\right)+k\right\}=\max _{j \neq i_{0}}\left\{v_{i_{0} \varepsilon}\left(x_{0}\right)-v_{j \varepsilon}\left(x_{0}\right)-k\right\} \leq-k .
$$

Since for every $i, v_{i}^{\varepsilon}$ is Lipschitz, we apply (10) and we have that

$$
v_{i_{0}}^{\varepsilon}\left(x_{0}\right)-\min _{j \neq i_{0}}\left\{v_{j}^{\varepsilon}\left(x_{0}\right)+k\right\} \leq-k+2 \varepsilon \max _{i}\left[v_{i}^{\varepsilon}\right]_{1},
$$

and, for all $y \in B\left(x_{0}, 2 \varepsilon\right)$,

$$
v_{i_{0}}^{\varepsilon}(y)-\min _{j \neq i_{0}}\left\{v_{j}^{\varepsilon}(y)+k\right\} \leq-k+4 \max _{i}\left[v_{i}^{\varepsilon}\right]_{1}\left(\varepsilon+\left|x_{0}-y\right|\right)<-k+12 \varepsilon \max _{i}\left[v_{i}^{\varepsilon}\right]_{1} .
$$

Taking $\varepsilon \leq\left(12 \max _{i}\left[v_{i}^{\varepsilon}\right]_{1}\right)^{-1} k$, obtain (38).

b) We prove (39). We claim that $v_{i_{0}}^{\varepsilon}(\cdot-e)$ is, for all $|e| \leq \varepsilon$, a viscosity super-solution of

$$
L^{\alpha_{i}}(x, D w(x))=0, \quad x \in B\left(x_{0}, \varepsilon\right) .
$$

Fix $e_{1} \in B(0, \varepsilon)$, and let $\varphi \in C^{2}\left(\mathbb{R}^{N}\right)$ be such that $v_{i_{0}}^{\varepsilon}\left(\cdot-e_{1}\right)-\varphi(\cdot)$ has a local minimum at $x_{e_{1}} \in B\left(x_{0}, \varepsilon\right)$. Then $v_{i_{0}}^{\varepsilon}(\cdot)-\varphi\left(\cdot+e_{1}\right)$ has a local minimum at $x_{e_{1}}-e_{1} \in B\left(x_{0}, 2 \varepsilon\right)$. Since $v^{\varepsilon}(\cdot)$ is a viscosity solution of (35), and $v_{i_{0}}^{\varepsilon}\left(x_{0}\right)-\min _{j \neq i_{0}}\left\{v_{j}^{\varepsilon}\left(x_{0}\right)+k\right\} \leq 0$ by (38), we have that

$$
\inf _{|e| \leq \varepsilon} L^{\alpha_{i_{0}}}\left(x_{e_{1}}-e_{1}+e, v_{i_{0}}^{\varepsilon}\left(x_{e_{1}}-e_{1}\right), D \varphi\left(x_{e_{1}}\right), D^{2} \varphi\left(x_{e_{1}}\right)\right) \geq 0, \quad \forall|e| \leq \varepsilon .
$$

In particular, for $e=e_{1}$, we obtain that $v_{i_{0}}^{\varepsilon}\left(\cdot-e_{1}\right)$ is a viscosity super-solution of (41). Since $e_{1}$ is an arbitrary point in $B(0, \varepsilon)$, we obtain that $v_{i_{0}}^{\varepsilon}(\cdot-e)$ is, for all $e \in B(0, \varepsilon)$, a viscosity 
super-solution of (41). Being a limit of convex combinations of $v_{i_{0}}^{\varepsilon}(\cdot-e)$, and a smooth function, $v_{i_{0} \varepsilon}(\cdot)$ is a classical super-solution on $(41)$, and hence $L^{\alpha_{i_{0}}}\left(x_{0}, \mathcal{D} v_{i_{0} \varepsilon}\left(x_{0}\right)\right) \geq 0$; relation (39) follows. $\square$

Define the two following sets:

$$
X:=\left\{x \in \mathbb{R}^{N} \mid u_{h}(x)=\psi(x)\right\} ; \quad Y:=\left\{x \in \mathbb{R}^{N} \mid S\left(h, x, u_{h},\left[u_{h}\right]_{x}\right)=0\right\} .
$$

Proposition 12 Under assumptions (A1), (A2) and (S1)-(S3), and assuming that (2) has a unique solution $u_{h}$ in $C_{b}\left(\mathbb{R}^{N}\right)$, we have that, if $x \in Y$, the following holds:

$$
u_{h}(x)-u(x) \leq C h^{\ell},
$$

where $\ell:=\min _{i \in J}\left\{k_{i} /(3 i-2)\right\}$ and $C$ depends only on $\lambda, K$ and $K_{c}$.

Proof. Consider the switching system (35), its solution $v^{\varepsilon}=\left(v_{1}^{\varepsilon}, \ldots, v_{M}^{\varepsilon}\right)$ and mollification $v_{\varepsilon}=\left(v_{1 \varepsilon}, \ldots, v_{M \varepsilon}\right)$. Let $w(y):=\min _{i} v_{i \varepsilon}(y)$. Define

$$
m:=\sup _{y \in Y}\left\{u_{h}(y)-w(y)\right\}=\sup _{i \in \mathcal{I}, y \in Y}\left\{u_{h}(y)-v_{i \varepsilon}(y)\right\} .
$$

Let $\phi(y):=\left(1+|y|^{2}\right)^{1 / 2}$. An approximation of $m$ is, for $k>0$, given by

$$
m_{k}:=\sup _{y \in Y}\left\{u_{h}(y)-w(y)-k \phi(y)\right\} .
$$

Since $u_{h}$ and $w$ are bounded, $\phi$ is coercive and $Y$ is a closed set, the supremum in (44) is attained at some point $x_{0} \in Y$. By the definition of $w$, we also have

$$
x_{0} \in \arg \max _{y \in Y}\left\{u_{h}(y)-v_{i_{0} \varepsilon}(y)-k \phi(y)\right\},
$$

when $i_{0} \in \arg \min _{j \in \mathcal{I}} v_{j \varepsilon}\left(x_{0}\right)$. In particular,

$$
m_{k} \geq u_{h}(y)-v_{i_{0} \varepsilon}(y)-k \phi(y), \quad \text { for all } y \in Y .
$$

Let $\varepsilon$ be such that (37) holds. Applying lemma 11, and since the first and the second order derivatives of $\phi$ are bounded, we have $\sup _{\alpha} L^{\alpha}\left(x_{0}, \mathcal{D}\left(v_{i_{0} \varepsilon}+k \phi\right)\left(x_{0}\right)\right) \geq-C k$. Combining with (S1), (S3), (44) and $x_{0} \in Y$, get

$$
\begin{aligned}
-C k-K_{C} Q\left(v_{i_{0} \varepsilon}+k \phi\right) & \leq S\left(h, x_{0},\left(v_{i_{0} \varepsilon}+k \phi\right)\left(x_{0}\right), v_{i_{0} \varepsilon}+k \phi\right) \\
& \leq S\left(h, x_{0}, u_{h}\left(x_{0}\right)-m_{k}, u_{h}-m_{k}\right) \\
& \leq-\lambda m_{k}+S\left(h, x_{0}, u_{h}\left(x_{0}\right), u_{h}\right)=-\lambda m_{k} .
\end{aligned}
$$

We obtain $\lambda m_{k} \leq K_{C} Q\left(v_{i_{0} \varepsilon}+k \phi\right)+C k$. Passing to the limit, get

$$
m \leq K_{C} Q\left(v_{i_{0} \varepsilon}\right) .
$$

INRIA 
In conclusion, we can say that for $x \in Y$ and for every $i \in \mathcal{I}$,

$$
\begin{aligned}
\sup _{y \in Y}\left\{u_{h}(y)-u(y)\right\} \leq & m+\sup _{y \in Y}\{w(y)-u(y)\} \\
\leq & m+\sup _{y \in Y}\left\{w(y)-v_{i \varepsilon}(y)\right\}+\sup _{y \in Y}\left\{v_{i \varepsilon}(y)-v_{i}^{\varepsilon}(y)\right\} \\
& +\sup _{y \in Y}\left\{v_{i}^{\varepsilon}(y)-v_{i}(y)\right\}+\sup _{y \in Y}\left\{v_{i}(y)-u(y)\right\} .
\end{aligned}
$$

Applying (36), (27), and the fact that $w(y) \leq v_{i \varepsilon}(y)$ for all $i \in \mathcal{I}$, we obtain

$$
\sup _{y \in Y}\left\{u_{h}(y)-u(y)\right\} \leq m+C \varepsilon+C k^{1 / 3} .
$$

where $C$ depends on $K, \lambda,[\psi]_{1}$ and $\max _{i}\left[v_{i}^{\varepsilon}\right]_{1}$. Using (47) and lemma 1 , we obtain

$$
u-u_{h} \leq K_{C} Q\left(v_{i_{0} \varepsilon}\right)+C \varepsilon+C k^{1 / 3}, \quad \forall x \in Y .
$$

The result follows by setting $\varepsilon=\max _{i \in J} h^{\frac{3 k_{i}}{3 i-2}}$ and using (37).

Theorem 13 Under assumptions (A1), (A2) and (S1)-(S3), and assuming that (2) has a unique solution $u_{h}$ in $C_{b, l}\left(\mathbb{R}^{N}\right)$, we have that

$$
u_{h}-u \leq C h^{\ell}, \quad \forall x \in \mathbb{R}^{N},
$$

where $\ell=\min _{i \in J}\left\{k_{i} /(3 i-2)\right\}$ and $C$ depends only on $\lambda, K$ and $K_{c}$.

Proof. If $x \in X$ we have that $u_{h}(x)=\psi(x) \leq u(x)$, therefore (50) holds. If $x \in Y$, then by theorem 4 , we have that $u_{h}(x)-u(x) \leq C h^{\ell}$. Since $X \cup Y=\mathbb{R}^{N}$, the conclusion follows.

\subsection{Extension to the case of a compact control set}

In this section we show that our results extend to the case of a precompact set of controls. We endow the set of controls with the distance $\mathrm{d}\left(\alpha, \alpha^{\prime}\right):=\left|\Phi^{\alpha}-\Phi^{\alpha^{\prime}}\right|_{0}$, where $\Phi^{\alpha}:=$ $\left(a^{\alpha}, b^{\alpha}, c^{\alpha}, f^{\alpha}\right)$. We suppose that $\sup _{\alpha \in \mathcal{A}}\left|\Phi^{\alpha}\right|_{1}<+\infty$. Precompactness of $\mathcal{A}$ is equivalent to the following condition:

(A3) for every $\delta>0$, there are $M \in \mathbb{N}$ and $\left\{\alpha_{i}\right\}_{i=1}^{M} \subset \mathcal{A}$, such that

$$
\sup _{\alpha \in \mathcal{A}} \inf _{1 \leq i \leq M}\left(\left|\sigma^{\alpha}-\sigma^{\alpha_{i}}\right|_{0}+\left|b^{\alpha}-b^{\alpha_{i}}\right|_{0}+\left|c^{\alpha}-c^{\alpha_{i}}\right|_{0}+\left|f^{\alpha}-f^{\alpha_{i}}\right|_{0}\right) \leq \delta .
$$

Consider the viscosity solution $u$ of

$$
\min \left\{\sup _{\alpha \in \mathcal{A}} L^{\alpha}(x, \mathcal{D} u(x)) ; u(x)-\psi(x)\right\}=0, \quad x \in \mathbb{R}^{N} .
$$


Existence, unicity and Lipschitzness of $u$ are proved in [11, lemma A.1]. Fix $\delta$ and consider $w_{\delta}$ the viscosity solution of

$$
\min \left\{\sup _{i \in \mathcal{I}_{M}} L^{\alpha_{i}}\left(x, \mathcal{D} w_{\delta}(x)\right) ; w_{\delta}(x)-\psi(x)\right\}=0, \quad x \in \mathbb{R}^{N},
$$

where $\mathcal{I}_{M}:=\{1, \ldots, M\}, M$ given by (A3). As in ([2, lemma 3.3]), we can show, by adapting the methods, that

$$
\left|u-w_{\delta}\right|_{0} \leq C \delta,
$$

where $C$ depends only on $K$ and $\lambda$. If we note $u_{h}$ the approximation of $u$ and $w_{h, \delta}$ the approximation of $w_{\delta}$, then we have $u_{h} \leq w_{h, \delta}$, and $w_{h, \delta}-w_{\delta} \leq C h^{\bar{\gamma}}$, where $\bar{\gamma}=\min _{i \in J}\left\{k_{i} /(3 i-2)\right\}$, $k_{i}$ given by (S3). From the proof of proposition 12, we can see that $C$ is independent of the size of $\mathcal{I}_{M}$. Then we have that

$$
-C h^{\gamma} \leq u_{h}-u \leq u_{h}-w_{h, \delta}+w_{h, \delta}-w+w-u \leq C h^{\bar{\gamma}}+C_{1} \delta
$$

where $\gamma=\min _{i \in J}\left\{k_{i} / i\right\}, \bar{\gamma}=\min _{i \in J}\left\{k_{i} /(3 i-2)\right\}, k_{i}$ given by (S3). All constants being independent of the size of $\mathcal{I}_{M}$, then we can choose $\delta$ of the order of $h^{\bar{\gamma}}$ and we obtain the same result as in theorem 13 .

Remark 14 It may happen that only $w_{h, \delta}$ is actually computed, and in that case it is useful to estimate $u-w_{h, \delta}$. Since $\left|u-w_{\delta}\right| \leq C \delta$, it follows from previous discussion that

$$
-C\left(\delta+h^{\gamma}\right) \leq w_{h, \delta}-u \leq C\left(\delta+h^{\bar{\gamma}}\right)
$$

\section{$5 \quad$ Specific approximation schemes}

In this section we apply our previous results to some specific discretization schemes.

\subsection{Finite differences, one dimensional problem}

Let $x$ be in $\mathbb{R}, \phi$ in $C^{n}(\mathbb{R}), h$ in $\mathbb{R}$ and define

$$
\delta_{ \pm} \phi(x)=\frac{\phi(x \pm h)-\phi(x)}{h}, \quad \Delta \phi(x)=\frac{\phi(x+h)-2 \phi(x)+\phi(x-h)}{h^{2}} .
$$

In particular, by a Taylor expansion, we obtain

$$
\left|\delta_{ \pm} \phi(x)-D \phi(x)\right| \leq \frac{1}{2} h\left|D^{2} \phi\right|, \quad\left|\Delta_{h} \phi(x)-D^{2} \phi(x)\right| \leq \frac{1}{12} h^{2}\left|D^{4} \phi\right| .
$$

Consider the finite difference scheme in $\mathbb{R}$ :

$$
\begin{aligned}
S(h, x, r, \phi) & := \\
& =\sup _{\alpha}\left\{-a^{\alpha}(x) \Delta \phi(x)-b_{+}^{\alpha}(x) \delta_{+} \phi(x)+b_{-}^{\alpha}(x) \delta_{-} \phi(x)+c^{\alpha}(x) r-f^{\alpha}(x)\right\},
\end{aligned}
$$

INRIA 
where $b_{+}^{\alpha}(x)=\max \left(b^{\alpha}(x), 0\right)$, and $b_{-}^{\alpha}(x)=\max \left(-b^{\alpha}(x), 0\right)$. For the consistency hypothesis (S3), we obtain, from the above Taylor expansion, $Q(\phi)=\left|D^{2} \phi\right| h+\left|D^{4} \phi\right| h^{2}$, i.e. $k_{2}=1$ and $k_{4}=2$. Then, by (30) and (50), we have

$$
-C h^{1 / 5} \leq u-u_{h} \leq C h^{1 / 2} .
$$

Remark 15 Consider a general scheme $S: \mathbb{R}^{+} \times \mathbb{R}^{N} \times \mathbb{R} \times C_{b}\left(\mathbb{R}^{N}\right) \rightarrow \mathbb{R}$, which satisfies (S1), (S2) and (S3), for some $k_{i}>0, i \in J$. To obtain equal or better estimate than (54) we must have:

$$
\min _{i \in J} \frac{k_{i}}{i} \geq \frac{1}{2}, \quad \min _{i \in J} \frac{k_{i}}{3 i-2} \geq \frac{1}{5} .
$$

In particular, the $k_{i}$ which give an equal or better estimate than (54) are

$$
\text { (i) } \mathrm{k}_{\mathrm{i}} \geq \mathrm{i} / 2, \text { for } \mathrm{i} \leq 4 ; \quad \text { (ii) } \mathrm{k}_{\mathrm{i}} \geq(3 \mathrm{i}-2) / 5 \text {, for } \mathrm{i} \geq 4 \text {. }
$$

Indeed, let $i \leq 4$. If $k_{i} \geq i / 2$, then we have also $k_{i} \geq(3 i-2) / 5$. Moreover, if $i>4$, we have $k_{i} \geq(3 i-2) / 5$ and also $k_{i} \geq i / 2$. Hence we obtain (55).

If the inequalities in (56) are strictly satisfied, then also the inequalities in (55) are strictly satisfied and we obtain a better estimate.

\subsection{Markov chain approximation}

The scheme (53) may be viewed as a particular Markov chain approximation of (1). We consider now a general Markov chain approximation of (1) in a regular grid, and we want to find conditions on the probabilities of transition to obtain estimate as in (54). We consider a discretization step $h \in \mathbb{R}$ and a regular grid of discretization $G^{h}$. With the coordinate $k=\left(k_{1}, \ldots, k_{N}\right)$ in $\mathbb{Z}^{N}$, is associated the point $x_{k} \in \mathbb{R}^{N}$ of the form $x_{k}:=\left(k_{1} h, \ldots, k_{N} h\right)$. Let $\left\{X_{q}^{h}, q \geq 0\right\}$ the states of the Markov chain at time $q$, with transition probabilities $p\left(x_{k}, y \mid \alpha\right), \alpha$ being the control value. Let $\Delta t^{h}$ an interpolation interval satisfying $\Delta t^{h} \rightarrow 0$ as $h \rightarrow 0$, and let $\mathbb{E}_{k, q}^{h, \alpha}$ be the conditional expectation of $X_{q+1}^{h}$, given $\left\{X_{q}^{h}=x_{k}\right\}$ and the control value $\alpha$. A possible adaptation for the cost function to this Markov chain is the following:

$$
W^{h}(x, \alpha)=\Delta t^{h}\left[\sum_{q \geq 0} f^{\alpha}\left(X_{q}^{h}\right)\left(1+c^{\alpha}(x) \Delta t^{h}\right)^{-q-1}\right] .
$$

Applying the dynamic programmic principle for the controlled chain $\left\{X_{q}^{h}, q \geq 0\right\}$, at state $x_{k} \in G_{h}$, we obtain the following relation:

$$
u_{h}\left(x_{k}\right)=\max \left\{\inf _{\alpha}\left(\frac{1}{1+c^{\alpha}\left(x_{k}\right) \Delta t^{h}}\left(\sum_{y} p\left(x_{k}, y \mid \alpha\right) u_{h}(y)+f^{\alpha}\left(x_{k}\right) \Delta t^{h}\right)\right) ; \psi\left(x_{k}\right)\right\} .
$$

Since $1+c^{\alpha}\left(x_{k}\right) \Delta t^{h} \geq 0$ for all $\alpha,(57)$ may be written in the form (2), with

$$
S\left(h, x_{k}, r, \phi\right)=\sup _{\alpha}\left\{-\frac{1}{\Delta t^{h}} \sum_{y} p\left(x_{k}, y \mid \alpha\right) \phi(y)-f^{\alpha}\left(x_{k}\right)+\frac{1}{\Delta t^{h}} r+c^{\alpha}\left(x_{k}\right) r\right\} .
$$


With the above definition for $S$, the assumptions (S1) and (S2) are satisfied. Suppose that (S3) is satisfied and we want to look for simple conditions on the probabilities $p\left(x_{k}, y\right)$ and on the $k_{i}$ defined in (S3), under those we obtain equal or better estimate than (54). We note $\mathbb{P}_{x, y}=\sum_{y} p(x, y \mid \alpha)$. Using remark 15 , we obtain the following:

Theorem 16 Let $S$ defined as in (58). Suppose that (S3) is satisfied for some $k_{i}, i \in J$.

(i) We have an equal or better estimate than (54) if and only if

$$
\begin{aligned}
& \text { (a1) }\left\|\frac{1}{\Delta t^{h}} \mathbb{P}_{x, y}(x-y)-b^{\alpha}(x)\right\|=K_{C} h^{k_{1}}, \\
& \text { (b1) }\left\|\frac{1}{2 \Delta t^{h}} \mathbb{P}_{x, y}(y-x)^{2}-a^{\alpha}(x)\right\|=K_{C} h^{k_{2}}, \\
& \text { (c1) }\left\|\frac{1}{i ! \Delta t^{h}} \mathbb{P}_{x, y}(y-x)^{i}\right\|=K_{C} h^{k_{i}}, \text { for } i=3,4,
\end{aligned}
$$

with

$$
k_{1} \geq \frac{1}{2}, k_{2} \geq 1, k_{3} \geq \frac{3}{2}, k_{4} \geq 2 .
$$

(ii) Moreover we have a better lower bound if and only if, in addition, $k_{4}$ satisfies strictly (59).

(iii) We have a better upper bound if and only if all the inequalities in (59) are strictly satisfied.

Proof. Fix $x \in \mathbb{R}$, and let $\phi \in C^{n}\left(\mathbb{R}^{N}\right)$, such that $D^{i} \phi$ is bounded for $i=1, \ldots, n$. Set $\Delta^{\phi}:=\left|\sup _{\alpha} L^{\alpha}(x, \mathcal{D} \phi(x))-S(h, x, \phi(x), \phi)\right|$. An upper bound of $\Delta^{\phi}$ is

$$
\left|\sup _{\alpha}\left(-\operatorname{tr}\left[a^{\alpha}(x) D^{2} \phi(x)\right]-b^{\alpha}(x) D \phi(x)+\frac{1}{\Delta t^{h}} \sum_{y} p(x, y \mid \alpha)(\phi(y)-\phi(x))\right)\right|
$$

From the Taylor expansion of $\phi(y)$ up to order 4 , we deduce that

$$
\Delta^{\phi} \leq \Delta_{1}^{\phi}+\Delta_{2}^{\phi}+\Delta_{3}^{\phi}+\Delta_{4}^{\phi}
$$

where

$$
\begin{aligned}
& \Delta_{1}^{\phi}:=\sup _{\alpha}\left|-b^{\alpha}(x) D \phi(x)+\frac{1}{\Delta t^{h}} \mathbb{P}_{x, y} D \phi(x)(y-x)\right|, \\
& \Delta_{2}^{\phi}:=\sup _{\alpha}\left|-\operatorname{tr}\left[a^{\alpha}(x) D^{2} \phi(x)\right]+\frac{1}{2 \Delta t^{h}} \mathbb{P}_{x, y} D^{2} \phi(x)(y-x)^{2}\right|, \\
& \Delta_{i}^{\phi}:=\sup _{\alpha}\left|\frac{1}{i ! \Delta t^{h}} \mathbb{P}_{x, y} D^{i} \phi(x)(y-x)^{i}\right|, \quad i=3,4 .
\end{aligned}
$$

INRIA 
Suppose now that conditions (a1)-(d1) and (59) are satisfied. Then $J=\{1,2,3,4\}$, and applying remark 15 , we obtain the result. Moreover, if $k_{4}>2$, then $k_{i} /(3 i-2)>1 / 5$ for all $i$. Hence we obtain a strictly better lower bound. Since $k_{i} / i \geq 1 / 2$ for all $i$ in $J$, if all $k_{i}$ satisfy strictly (59), we have a better upper bound.

Suppose now that we have a better or equal estimate than (54). Then we have

$$
\Delta^{\phi} \leq K_{C} \sum_{i \in J}\left|D^{i} \phi\right| h^{k_{i}}
$$

with $\min _{i \in J} k_{i} / i \geq 1 / 2$ and $\min _{i \in J} k_{i} /(3 i-2) \geq 1 / 5$, and here $J=\{1,2,3,4\}$. From (61), (60) and remark 17, we have that (a1)-(d1) are satisfied with $k_{i}$ as in (59). If the lower bound is strictly bigger that $1 / 5$, since $k_{i} /(3 i-2)>1 / 5$ for $i=1,2,3$, then we must have $k_{4}>2$. If the upper bound is strictly bigger than $1 / 2$, since $k_{i} \geq i / 2$ for all $i$, then we must have $k_{i}>i / 2$ for all $i$.

Remark 17 (i) We have that conditions (a1) and (b1) imply the consistence in the sense of Kushner (see [15]), i.e.

$$
\left\|\mathbb{E}(y-x)-b^{\alpha}(x) \Delta t^{h}\right\| \leq \Delta t^{h} r_{1}, \quad\left\|\operatorname{Cov}(y)-2 a^{\alpha}(x)\right\| \leq \Delta t^{h} r_{2} .
$$

In [15] we have $r_{i}=o(1)$, for $i=1,2$. Our error estimate need the more restrictive conditions $r_{1}=h^{k_{1}}$ and $r_{2}=h^{k_{2}}+\Delta t^{h} h^{2 k_{1}}+\Delta t^{h}$.

(ii) We remark that to obtain (a1)-(d1), we use the inequality

$$
\Delta_{i}^{\phi} \leq\left|D^{i} \phi\right| \cdot\left\|\mathbb{E}(y-x)^{i}-a^{i}\right\|,
$$

for some $a^{i}$ and for all $\phi$. This inequality is sharp, since $\left\|\mathbb{E}(y-x)^{i}-a^{i}\right\|$ is the optimal constant for which we have this upper bound (for any function $\phi$ ). Indeed, let $B$ an i-linear symmetric form. The optimal constant $C$ for which

$$
\left|D^{i} \phi(x) B\right| \leq C\left|D^{i} \phi\right|, \forall \phi \in C^{n}\left(\mathbb{R}^{N}\right) \text { such that } D^{i} \phi \text { is bounded } \forall i,
$$

is $C=|B|$. Indeed, we may identify $a^{i}$ and $\mathbb{E}(y-x)^{i}$ with $i$-linear symmetric forms, and the above display reduces to the Cauchy-Schwarz inequality for $i$-linear symmetric forms.

(iii) Let $\Delta^{\phi}:=\left|\sum_{i=1}^{4} D^{i} \phi(x) \mathbb{E}(y-x)^{i}-a^{i} D^{i} \phi(x)\right|$, for some $a^{i}$. We have that the optimal constants $C_{i}$ such that

$$
\Delta \leq \sum_{i=1}^{4} C_{i} \mid D^{i} \phi\left(\mathbb{E}(y-x)^{i}-a^{i} \mid, \forall \phi \in C^{n}\left(\mathbb{R}^{N}\right) \text { such that } D^{i} \phi \text { is bounded } \forall i,\right.
$$

are $C_{i}=1$, for all $i$. 


\subsection{A counter example}

We give here an example of a finite difference scheme for which the $k_{i}$ do not satisfy conditions given in remark 15, and we will show that we obtain a estimate worse than (54). Consider the following equation

$$
\sup _{\alpha}\left\{-\operatorname{tr}\left[a^{\alpha}(x) D^{2} u(x)\right]+c^{\alpha}(x) u(x)-f^{\alpha}(x)\right\}=0, \quad x \in \mathbb{R}^{2},
$$

with

$$
b^{\alpha}(x)=0, a^{\alpha}(x)=\left(\begin{array}{cc}
1 & 0 \\
0 & 2
\end{array}\right), \quad \forall x, \alpha .
$$

Let $h$ the discretization step and $\Delta t^{h}$ the interpolation interval. We consider the following probabilities of transition:

$$
p\left(x, x-h e_{2} \mid \alpha\right)=\frac{1}{2} ; \quad p\left(x, x \pm h e_{1}+h e_{2} \mid \alpha\right)=\frac{1}{4} .
$$

In particular, if we choose $\Delta t^{h}=\frac{1}{4} h^{2}$, we have that these probabilities verify

$$
\mathbb{E}(y-x)=\frac{1}{2}\left(\begin{array}{c}
0 \\
-h
\end{array}\right)+\frac{1}{4}\left(\begin{array}{c}
h \\
h
\end{array}\right)+\frac{1}{4}\left(\begin{array}{c}
-h \\
h
\end{array}\right)=\left(\begin{array}{l}
0 \\
0
\end{array}\right),
$$

and

$$
\begin{aligned}
\mathbb{E}(y-x)^{2} & =\frac{1}{2}\left(\begin{array}{cc}
0 & 0 \\
0 & h^{2}
\end{array}\right)+\frac{1}{4}\left(\begin{array}{cc}
h^{2} & h^{2} \\
h^{2} & h^{2}
\end{array}\right)+\frac{1}{4}\left(\begin{array}{cc}
h^{2} & -h^{2} \\
-h^{2} & h^{2}
\end{array}\right) \\
& =\Delta t^{h}\left(\begin{array}{ll}
1 & 0 \\
0 & 2
\end{array}\right) .
\end{aligned}
$$

We have that

$$
\begin{aligned}
& \sum_{y} \frac{p(x, y)}{h^{2}} D^{3} \phi(x)(y-x)^{3} \\
& =\sum_{y} \frac{p(x, y)}{h^{2}}\left[\sum_{i=0}^{3} \frac{\partial^{3} \phi}{\partial x_{1}^{i} \partial x_{2}^{3-i}}(x)\left(y_{1}-x_{1}\right)^{i}\left(y_{2}-x_{2}\right)^{3-i}\right] \\
& =-\frac{1}{2} \frac{\partial^{3} \phi}{\partial x_{2}^{3}}(x) h+\frac{1}{4}\left(\frac{\partial^{3} \phi}{\partial x_{1}^{3}}(x) h+\frac{\partial^{3} \phi}{\partial x_{1}^{2} x_{2}}(x) h+\frac{\partial^{3} \phi}{\partial x_{1} x_{2}^{2}}(x) h+\frac{\partial^{3} \phi}{\partial x_{2}^{3}}(x) h\right) \\
& \quad+\frac{1}{4}\left(-\frac{\partial^{3} \phi}{\partial x_{1}^{3}}(x) h+\frac{\partial^{3} \phi}{\partial x_{1}^{2} x_{2}}(x) h-\frac{\partial^{3} \phi}{\partial x_{1} x_{2}^{2}}(x) h+\frac{\partial^{3} \phi}{\partial x_{2}^{3}}(x) h\right) \\
& =\frac{1}{2} \frac{\partial^{3} \phi}{\partial x_{1}^{2} x_{2}}(x) h .
\end{aligned}
$$

Hence we can write (S3) in the following way

$$
\left|\sup _{\alpha} L^{\alpha}\left(x, \phi(x), D \phi(x), D^{2} \phi(x)\right)-S(x, h, \phi(x), \phi)\right| \leq
$$

INRIA 


$$
\frac{1}{2}\left|\frac{\partial^{3} \phi}{\partial x_{1}^{2} \partial x_{2}}(x)\right| h+\left(\frac{1}{2}\left|\frac{\partial^{4} \phi}{\partial x_{1}^{4}}(x)\right|+\frac{1}{2}\left|\frac{\partial^{4} \phi}{\partial x_{1}^{2} \partial x_{2}^{2}}\right|(x)+\left|\frac{\partial^{4} \phi}{\partial x_{2}^{4}}\right|(x)\right) h^{2} .
$$

So, we have $k_{3}=1$ and $k_{4}=2$, and by applying theorem 3 and theorem 4 we obtain

$$
-C h^{1 / 7} \leq u-u_{h} \leq C h^{1 / 3} .
$$

\subsection{The generalized finite differences scheme}

We consider the generalized finite differences scheme defined in [5]. Let $\varphi=\left\{\varphi_{k}\right\}$ be a real valued function over $\mathbb{Z}^{N}$. Let $\xi \in \mathbb{Z}^{N}$ and consider the finite difference operator

$$
\Delta_{\xi} \varphi_{k}:=\varphi_{k+\xi}+\varphi_{k-\xi}-2 \varphi_{k} .
$$

In $\phi$ belongs to $C^{2}\left(\mathbb{R}^{N}\right)$, and $\varphi_{k}:=\phi\left(x_{k}\right)$ for all $k$, then we have

$$
\Delta_{\xi} \varphi_{k}:=\phi\left(x_{k+\xi}\right)+\phi\left(x_{k-\xi}\right)-2 \phi\left(x_{k}\right) .
$$

Then we consider

$$
\left(D_{k} u_{h}\left(x_{k}\right)\right)_{i}= \begin{cases}\frac{u_{h}\left(x_{k+e_{i}}\right)-u_{h}\left(x_{k}\right)}{h} & \text { if } b_{i}^{\alpha}\left(x_{k}\right) \geq 0, \\ \frac{u_{h}\left(x_{k}\right)-u_{h}\left(x_{k-e_{i}}\right)}{h} & \text { if } b_{i}^{\alpha}\left(x_{k}\right) \leq 0 .\end{cases}
$$

Let $\mathcal{S}$ be a finite set of $\mathbb{Z} \backslash\{0\}$ containing $\left\{e_{1}, \ldots, e_{N}\right\}$. We consider the following probabilities of transition

$$
\begin{aligned}
& p^{\alpha}\left(x_{k}, x_{k} \mid \alpha\right)=1-\Delta t^{h} \sum_{i=1}^{N}\left(\frac{\left|b_{i}^{\alpha}\left(x_{k}\right)\right|}{h}+2 \sum_{\xi \in \mathcal{S}} \alpha_{k, \xi}\right), \\
& p^{\alpha}\left(x_{k}, x_{k} \pm e_{i} h \mid \alpha\right)=\Delta t^{h}\left(\frac{b_{i}^{\alpha \pm}\left(x_{k}\right)}{h}+\alpha_{k, e_{i}}\right), \\
& p^{\alpha}\left(x_{k}, x_{k}+\xi h \mid \alpha\right)=\Delta t^{h} \alpha_{k, \xi} \quad \text { for } \xi \in \mathcal{S}, \xi \neq e_{i}, \\
& p^{\alpha}\left(x_{k}, y \mid \alpha\right)=0 \text { for } y \notin x_{k+\mathcal{S}} .
\end{aligned}
$$

Then we can write (58) in the following way:

$$
S\left(h, x_{k}, r, \phi\right)=\sup _{\alpha}\left\{-\sum_{\xi \in \mathcal{S}} \alpha_{k, \xi} \Delta_{\xi} \phi\left(x_{k}\right)-b^{\alpha}\left(x_{k}\right) D_{k} \phi\left(x_{k}\right)+c^{\alpha}\left(x_{k}\right) r-f^{\alpha}\left(x_{k}\right)\right\} .
$$

We make the strong consistency hypothesis on the matrix

$$
a^{\alpha}(x)=\sum_{i, j} h^{2} \xi_{i} \xi_{j} \alpha_{k, \xi} e_{i} e_{j}^{T}, \quad \forall k \in \mathbb{Z}^{N} .
$$

The scheme defined in (65) satisfies (S1) et (S2). We consider a function $\phi \in C^{2}\left(\mathbb{R}^{N}\right)$. By applying a Taylor expansion, we obtain 
(S3) $\left|\sup _{\alpha \in \mathcal{A}} L^{\alpha}\left(x, \phi, D \phi, D^{2} \phi\right)-S(x, h, \phi(x), \phi)\right| \leq$ $\sup _{\alpha \in \mathcal{A}}\left|b^{\alpha}\right|_{0}\left|D^{2} \phi\right|_{0} h+\sup _{\alpha \in \mathcal{A}}\left|\sigma^{\alpha}\right|_{0}^{2}\left|D^{4} \phi\right|_{0} h^{2}$.

So we can say that $k_{2}=1$ and $k_{4}=2$. Applying theorems 3 and 4 , obtain the same estimate as in the case of one player (see [2]).

Theorem 18 Assume (A1)-(A4), (S1)-(S3). If $u$ and $u_{h}$ are solution of (1) and (2), with $S$ defined as in (65), then for $h$ sufficiently small we obtain

$$
-C h^{1 / 5} \leq u-u_{h} \leq C h^{1 / 2} .
$$

\section{A Well-posedness of the switching system}

In this appendix we prove the well-posedness of the switching system (12), for $k \geq 0$, under assumptions (A1) and (A2) on the coefficients (stated in section 2). Well-posedness of the original equation (1) will follow by setting $k=0$. Let us start by stating a technical lemma which is an easy extension of ([2, lemma A.2]):

Lemma 19 Let $v$ be a bounded and continuous sub-solution on (12) and $\bar{v}$ be a bounded and continuous super-solution of another equation (12), where $L^{\alpha}$ is replaced by $\bar{L}^{\alpha}$, satisfying the same assumptions with coefficients $\left(\bar{\sigma}^{\alpha}, \bar{b}^{\alpha}, \bar{c}^{\alpha}, \bar{f}^{\alpha}\right)$. Let $g \in C^{2}\left(\mathbb{R}^{N} \times \mathbb{R}^{N}\right)$. Consider

$$
m:=\sup _{i, x, y}\left\{v_{i}(x)-\bar{v}_{i}(y)-g(x, y)\right\},
$$

and suppose that the "sup" is attained at some point $\left(i_{0}, x_{0}, y_{0}\right)$. Set

$$
A:=\left\{i \in \mathcal{I} \mid\left(i, x_{0}, y_{0}\right) \text { realizes the sup }\right\} ; I\left(x_{0}\right):=\left\{i \in \mathcal{I} \mid v_{i}\left(x_{0}\right) \leq \psi\left(x_{0}\right)\right\} .
$$

If $A \cap I\left(x_{0}\right)=\varnothing$, then there exists $i \in A$ such that

$$
\bar{v}_{i}\left(y_{0}\right)<\min _{j \neq i}\left\{\bar{v}_{j}\left(y_{0}\right)+k\right\} .
$$

Proof. We proceed by contradiction. Let $j$ in $A$. If (66) does not hold, there exists $\ell \in \mathcal{I}$ such that

$$
\bar{v}_{j}\left(y_{0}\right) \geq \bar{v}_{l}\left(y_{0}\right)+k .
$$

Since $A \cap I\left(x_{0}\right)=\varnothing$, then for all $i \in A$,

$$
\max \left\{L^{\alpha_{i}}\left(x_{0}, v_{i}\left(x_{0}\right), D_{x} g\left(x_{0}\right), D_{x x}^{2} g\left(x_{0}\right)\right) ; v_{i}\left(x_{0}\right)-\min _{j \neq i}\left\{v_{j}\left(x_{0}\right)+k\right\}\right\} \leq 0 .
$$

Hence we obtain $v_{j}\left(x_{0}\right) \leq v_{l}\left(x_{0}\right)+k$, and then with (67),

$$
v_{j}\left(x_{0}\right)-\bar{v}_{j}\left(y_{0}\right) \leq v_{l}\left(x_{0}\right)-\bar{v}_{l}\left(y_{0}\right) .
$$

INRIA 
Therefore $l \in A$, and equality holds in (68). Then $\bar{v}_{j}\left(y_{0}\right)=\bar{v}_{l}\left(y_{0}\right)+k$. Since $A$ is a finite set, this proves that there exists a finite sequence $j_{1}, \ldots, j_{K} \in A$ such that $\bar{v}_{j_{i}}\left(y_{0}\right)=\bar{v}_{j_{i+1}}\left(y_{0}\right)+k$ for $i=1, \ldots, K-1$, and $j_{1}=j_{K}$. So we obtain

$$
0=\sum_{i=1}^{K-1}\left(\bar{v}_{j_{i}}\left(y_{0}\right)-\bar{v}_{j_{i+1}}\left(y_{0}\right)\right)=(K-1) k>0,
$$

and we have a contradiction. Therefore (66) holds.

Now we can state the following lemma about comparison, existence, uniqueness and the bounds on the solution $v=\left(v_{1}, \ldots, v_{M}\right)$ of $(12)$. This is an easy extension of $([2$, theorem A.1]).

Lemma 20 Under assumptions (A1) and (A2), the following statements hold:

(a) If $v$ and $w$ are respectively sub-solution and super-solution of (12), such that $v_{i}, w_{i} \in$ $C_{b}\left(\mathbb{R}^{N}\right)$ for all $i \in \mathcal{I}$, then $v \leq w$ in $\mathbb{R}^{N}$.

(b) There exists a unique viscosity solution $v$ of (12), such that $v_{i} \in C_{b, l}\left(\mathbb{R}^{N}\right)$ for all $i \in \mathcal{I}$. This solution satisfies

$$
\begin{aligned}
& \max _{i}\left|v_{i}\right|_{0} \leq \max \left\{\lambda^{-1} \sup _{\alpha}\left|f^{\alpha}\right|_{0} ;|\psi|_{0}\right\}, \\
& \max _{i}\left[v_{i}\right]_{1} \leq \max \left\{\sup _{i, \alpha} \frac{\left[c^{\alpha}\right]_{1}\left|v_{i}\right|_{0}+\left[f^{\alpha}\right]_{1}}{\min \{1, \lambda\}-\left[\sigma^{\alpha}\right]_{1}^{2}-\left[b^{\alpha}\right]_{1}} ;[\psi]_{1}\right\} .
\end{aligned}
$$

Proof. (a) This is a consequence of the comparison principle [10, theorem 3.1].

(b) Existence and uniqueness follow from the comparison principle; they are proved in [10] for the Dirichlet problem on a bounded domain. To extend the result to an unbounded domain, we have only to modify the test functions of [10] in the standard way. Let $M:=$ $\max \left\{\sup _{\alpha} \lambda^{-1}\left|f^{\alpha}\right|_{0} ;|\psi|_{0}\right\}$. It is easy to see that $M$ and $-M$ are respectively super and subsolution of (12). Hence, by the comparison principle we obtain the bound on $\max _{i}\left|v_{i}\right|_{0}$. To obtain the bound on $\max _{i}\left[v_{i}\right]_{1}$, we set

$$
m:=\sup _{i, x, y} \phi_{i}(x, y):=\sup _{i, x, y}\left\{v_{i}(x)-v_{i}(y)-\delta|x-y|^{2}-\varepsilon\left(|x|^{2}+|y|^{2}\right)\right\},
$$

where $\delta>0$ and $\varepsilon>0$. The supremum is attained at a point $\left(i_{0}, x_{0}, y_{0}\right)$, so

$$
m=v_{i_{0}}\left(x_{0}\right)-v_{i_{0}}\left(y_{0}\right)-\delta\left|x_{0}-y_{0}\right|^{2}-\varepsilon\left(\left|x_{0}\right|^{2}+\left|y_{0}\right|^{2}\right) .
$$

Define the following sets:

$$
A:=\left\{i \in \mathcal{I} \mid\left(i, x_{0}, y_{0}\right) \text { realizes the } \sup \right\}, I\left(x_{0}\right):=\left\{i \in \mathcal{I} \mid v_{i}\left(x_{0}\right)=\psi\left(x_{0}\right)\right\} .
$$


The maximum principle for semi-continuous functions (see [7]), and the definition of viscosity solutions imply that, for $i \in A$, there exist $X, Y \in \mathcal{S}^{N}$ such that

$$
\begin{gathered}
\min \left\{\max \left(L^{\alpha_{i}}\left(x_{0}, v_{i}\left(x_{0}\right), p_{x}, X\right) ; v_{i}\left(x_{0}\right)-\min _{j \neq i}\left\{v_{j}\left(x_{0}\right)+k\right\}\right) ; v_{i}\left(x_{0}\right)-\psi\left(x_{0}\right)\right\} \leq 0, \\
\min \left\{\max \left(L^{\alpha_{i}}\left(y_{0}, v_{i}\left(y_{0}\right), p_{y}, Y\right) ; v_{i}\left(y_{0}\right)-\min _{j \neq i}\left\{v_{j}\left(y_{0}\right)+k\right\}\right) ; v_{i}\left(y_{0}\right)-\psi\left(y_{0}\right)\right\} \geq 0,
\end{gathered}
$$

where $p_{x}=2 \delta\left(x_{0}-y_{0}\right)+2 \varepsilon x_{0}, p_{y}=2 \delta\left(x_{0}-y_{0}\right)-2 \varepsilon y_{0}$, and there exists $\ell>0$ such that

$$
\left(\begin{array}{cc}
X & 0 \\
0 & -Y
\end{array}\right) \leq \ell \delta\left(\begin{array}{cc}
I & -I \\
-I & I
\end{array}\right)+\ell \varepsilon\left(\begin{array}{cc}
I & 0 \\
0 & I
\end{array}\right)+\mathcal{O}(k)
$$

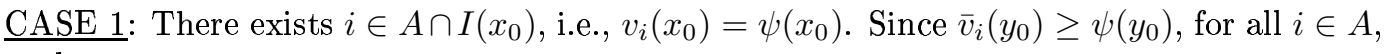
we have

$$
v_{i}\left(x_{0}\right)-\bar{v}_{i}\left(y_{0}\right) \leq \psi\left(x_{0}\right)-\psi\left(y_{0}\right) \leq[\psi]_{1}\left|x_{0}-y_{0}\right| .
$$

CASE 2: The set $A \cap I\left(x_{0}\right)$ is empty. Then

$$
\max \left\{L^{\alpha_{i}}\left(x_{0}, v_{i}\left(x_{0}\right), p_{x}, X\right) ; v_{i}\left(x_{0}\right)-\min _{j \neq i}\left\{v_{j}\left(x_{0}\right)+k\right\}\right\} \leq 0, \quad \forall i \in A .
$$

Since $\max \left\{L^{\alpha_{i_{0}}}\left(y_{0}, v_{i_{0}}\left(y_{0}\right), p_{y}, Y\right) ; v_{i_{0}}\left(y_{0}\right)-\min _{j \neq i_{0}}\left\{v_{j}\left(y_{0}\right)+k\right\}\right\} \geq 0$, applying lemma 19, obtain

$$
L^{\alpha_{i_{0}}}\left(x_{0}, v_{i_{0}}\left(x_{0}\right), p_{x}, X\right) \leq 0 \leq L^{\alpha_{i_{0}}}\left(y_{0}, v_{i_{0}}\left(y_{0}\right), p_{y}, Y\right) .
$$

Now we can proceed as in the standard situation (see [12, lemma A.1]).

Combining cases 1 and 2 we obtain the result.

By using ([1, theorem A.1]), we prove the following theorem.

Theorem 21 Let $v$ and $\bar{v}$ be solutions of (12) with coefficients $\sigma, b, c, f$ and $\bar{\sigma}, \bar{b}, \bar{c}, \bar{f}$ respectively. Suppose that assumptions (A1), (A2) are satisfied for both sets of coefficients with the same $\lambda$, and $\max _{i}\left|v_{i}\right|_{1}+\max _{i}\left|\bar{v}_{i}\right|_{1} \leq M<\infty$. Then

$$
\lambda \max _{i}\left|v_{i}-\bar{v}_{i}\right|_{0} \leq M\left(\sup _{\alpha}\left\{\left|\sigma^{\alpha}-\bar{\sigma}^{\alpha}\right|_{0}+\left|b^{\alpha}-\bar{b}^{\alpha}\right|_{0}+\left|c^{\alpha}-\bar{c}^{\alpha}\right|_{0}+\left|f^{\alpha}-\bar{f}^{\alpha}\right|_{0}\right\}\right),
$$

where $M$ depends on $K, \sup _{i}\left|v_{i}\right|_{0}, \sup _{i}\left|\bar{v}_{i}\right|_{0}$.

Proof. We set

$$
m:=\sup _{i, x, y} \phi_{i}(x, y):=\sup _{i, x, y}\left\{v_{i}(x)-\bar{v}_{i}(y)-\delta|x-y|^{2}-\varepsilon\left(|x|^{2}+|y|^{2}\right)\right\},
$$

where $\delta>0$ and $\varepsilon>0$. The "sup" is attained at a point $\left(i, x_{0}, y_{0}\right)$, so

$$
m=v_{i}\left(x_{0}\right)-\bar{v}_{i}\left(y_{0}\right)-\delta\left|x_{0}-y_{0}\right|^{2}-\varepsilon\left(\left|x_{0}\right|^{2}+\left|y_{0}\right|^{2}\right) .
$$

INRIA 
Let

$$
A:=\left\{i \in \mathcal{I} \mid\left(i, x_{0}, y_{0}\right) \text { realize the } \sup \right\}, \quad I\left(x_{0}\right):=\left\{i \in \mathcal{I} \mid v_{i}\left(x_{0}\right)=\psi\left(x_{0}\right)\right\} .
$$

The maximum principle for semi-continuous functions (see [7]), and the definition of viscosity solutions imply that, for $i \in A$, there exist $X, Y \in \mathcal{S}^{N}$ such that

$$
\begin{aligned}
& \min \left\{\max \left(L^{\alpha_{i}}\left(x_{0}, v_{i}\left(x_{0}\right), p_{x}, X\right) ; v_{i}\left(x_{0}\right)-\min _{j \neq i}\left\{v_{j}\left(x_{0}\right)+k\right\}\right) ; v_{i}\left(x_{0}\right)-\psi\left(x_{0}\right)\right\} \leq 0, \\
& \min \left\{\max \left(L^{\alpha_{i}}\left(y_{0}, \bar{v}_{i}\left(y_{0}\right), p_{y}, Y\right) ; \bar{v}_{i}\left(y_{0}\right)-\min _{j \neq i}\left\{\bar{v}_{j}\left(y_{0}\right)+k\right\}\right) ; \bar{v}_{i}\left(y_{0}\right)-\psi\left(y_{0}\right)\right\} \geq 0,
\end{aligned}
$$

where $p_{x}=2 \delta\left(x_{0}-y_{0}\right)+2 \varepsilon x_{0}, p_{y}=2 \delta\left(x_{0}-y_{0}\right)-2 \varepsilon y_{0}$, and there exists $\ell>0$ such that

$$
\left(\begin{array}{cc}
X & 0 \\
0 & -Y
\end{array}\right) \leq \ell \delta\left(\begin{array}{cc}
I & -I \\
-I & I
\end{array}\right)+\ell \varepsilon\left(\begin{array}{cc}
I & 0 \\
0 & I
\end{array}\right)+\mathcal{O}(k)
$$

We have to study two different cases.

CASE 1: If there exists $i \in A \cap I\left(x_{0}\right)$, then $v_{i}\left(x_{0}\right)=\psi\left(x_{0}\right)$. Since $\bar{v}_{i}\left(y_{0}\right) \geq \psi\left(y_{0}\right)$, then we have

$$
v_{i}\left(x_{0}\right)-\bar{v}_{i}\left(y_{0}\right) \leq \psi\left(x_{0}\right)-\psi\left(y_{0}\right) \leq[\psi]_{1}\left|x_{0}-y_{0}\right| .
$$

CASE 2: If $A \cap I\left(x_{0}\right)=\varnothing$, then

$$
\max \left\{L^{\alpha_{i}}\left(x_{0}, v_{i}\left(x_{0}\right), p_{x}, X\right) ; v_{i}\left(x_{0}\right)-\min _{j \neq i}\left\{v_{j}\left(x_{0}\right)+k\right\}\right\} \leq 0, \quad \forall i \in A .
$$

Since, $\max \left\{L^{\alpha_{i_{0}}}\left(y_{0}, \bar{v}_{i_{0}}\left(y_{0}\right), p_{y}, Y\right) ; \bar{v}_{i_{0}}\left(y_{0}\right)-\min _{j \neq i_{0}}\left\{\bar{v}_{j}\left(y_{0}\right)+k\right\}\right\} \geq 0$, applying lemma 19, we obtain

$$
L^{\alpha_{i_{0}}}\left(x_{0}, v_{i_{0}}\left(x_{0}\right), p_{x}, X\right) \leq 0 \leq \bar{L}^{\alpha_{i_{0}}}\left(y_{0}, \bar{v}_{i_{0}}\left(y_{0}\right), p_{y}, Y\right),
$$

and then

$$
\begin{gathered}
0 \leq-\operatorname{tr}\left[\bar{a}^{\alpha_{i_{0}}}\left(y_{0}\right) Y\right]+\operatorname{tr}\left[a^{\alpha_{i_{0}}}\left(x_{0}\right) X\right]-\bar{b}^{\alpha_{i_{0}}}\left(y_{0}\right) p_{y}+b^{\alpha_{i_{0}}}\left(x_{0}\right) p_{x}+ \\
\bar{c}^{\alpha_{i_{0}}}\left(y_{0}\right) \bar{v}_{i_{0}}\left(y_{0}\right)-c^{\alpha_{i_{0}}}\left(x_{0}\right) v_{i_{0}}\left(x_{0}\right)-\bar{f}^{\alpha_{i_{0}}}\left(y_{0}\right)+f^{\alpha_{i_{0}}}\left(x_{0}\right) \\
=:(I)+(I I)+(I I I)+(I V) .
\end{gathered}
$$

As in [1], we analyze each term separately:

$$
\begin{aligned}
(I)= & \operatorname{tr}\left[a^{\alpha_{i_{0}}}\left(x_{0}\right) X\right]-\operatorname{tr}\left[\bar{a}^{\alpha_{i_{0}}}\left(y_{0}\right) Y\right] \\
\leq & 2 \ell \delta\left\{\left|\sigma^{\alpha_{i_{0}}}\left(x_{0}\right)-\bar{\sigma}^{\alpha_{i_{0}}}\left(x_{0}\right)\right|^{2}+\left|\bar{\sigma}^{\alpha_{i_{0}}}\left(x_{0}\right)-\bar{\sigma}^{\alpha_{i_{0}}}\left(y_{0}\right)\right|^{2}\right\} \\
& +\ell \varepsilon\left\{\left|\sigma^{\alpha_{i_{0}}}\left(x_{0}\right)\right|^{2}+\left|\bar{\sigma}^{\alpha_{i_{0}}}\left(y_{0}\right)\right|^{2}\right\}, \\
(I I)= & \left(b^{\alpha_{i_{0}}}\left(x_{0}\right)-\bar{b}^{\alpha_{i_{0}}}\left(y_{0}\right)\right)\left(x_{0}-y_{0}\right) \\
\leq & 2\left|b^{\alpha_{i_{0}}}\left(x_{0}\right)-\bar{b}^{\alpha_{i_{0}}}\left(x_{0}\right)\right|^{2}+2\left|x_{0}-y_{0}\right|^{2} \\
& +\left|\bar{b}^{\alpha_{i_{0}}}\left(x_{0}\right)-\bar{b}^{\alpha_{i_{0}}}\left(y_{0}\right)\right|\left|x_{0}-y_{0}\right|, \\
(I I I)= & \bar{c}^{\alpha_{i_{0}}}\left(y_{0}\right) \bar{u}\left(y_{0}\right)-c^{\alpha_{i_{0}}}\left(x_{0}\right) u\left(x_{0}\right) \\
\leq & \left|u\left(x_{0}\right)\right|\left|c^{\alpha_{i_{0}}}\left(x_{0}\right)-\bar{c}^{\alpha_{i_{0}}}\left(x_{0}\right)\right|+\left|\bar{u}\left(y_{0}\right)\right|\left|\bar{c}^{\alpha_{i_{0}}}\left(x_{0}\right)-\bar{c}^{\alpha_{i_{0}}}\left(y_{0}\right)\right| \\
& -\lambda m, \\
(I V)= & f^{\alpha_{i_{0}}}\left(x_{0}\right)-\bar{f}^{\alpha_{i_{0}}}\left(y_{0}\right) \\
\leq & \left|f^{\alpha_{i_{0}}}\left(x_{0}\right)-\bar{f}^{\alpha_{i_{0}}}\left(x_{0}\right)\right|+\left|\bar{f}^{\alpha_{i_{0}}}\left(x_{0}\right)-\bar{f}^{\alpha_{i_{0}}}\left(y_{0}\right)\right|\left|x_{0}-y_{0}\right| .
\end{aligned}
$$

$\mathrm{RR} \mathrm{n}^{\circ} 5441$ 
Hence we obtain

$$
\begin{gathered}
\lambda m \leq 2 \ell \delta\left\{\left|\sigma^{\alpha_{i_{0}}}-\bar{\sigma}^{\alpha_{i_{0}}}\right|_{0}^{2}+\left|b^{\alpha_{i_{0}}}-\bar{b}^{\alpha_{i_{0}}}\right|_{0}^{2}\right\}+ \\
+\left\{\left|v_{i_{0}}\right|_{0}\left|\bar{c}^{\alpha_{i_{0}}}-c^{\alpha_{i_{0}}}\right|_{0}+\left|f^{\alpha_{i_{0}}}-\bar{f}^{\alpha_{i_{0}}}\right|_{0}\right\}+ \\
+2 \delta\left\{\ell\left[\bar{\sigma}^{\alpha_{i_{0}}}\right]_{1}^{2}+\left[\bar{b}^{\alpha_{i_{0}}}\right]_{1}+2\right\}\left|x_{0}-y_{0}\right|^{2}+ \\
\left\{\left|\bar{v}_{i_{0}}\right|_{0}\left[\bar{c}^{\alpha_{i_{0}}}\right]_{1}+\left[\bar{f}^{\alpha_{i_{0}}}\right]_{1}\right\}\left|x_{0}-y_{0}\right|+C \varepsilon\left(1+\left|x_{0}\right|+\left|y_{0}\right|\right) .
\end{gathered}
$$

We sum the bounds obtained in the two cases to have a general bound of $m$. So we obtain

$$
\begin{gathered}
\lambda m \leq 2 \ell \delta\left\{\left|\sigma^{\alpha_{i_{0}}}-\bar{\sigma}^{\alpha_{i_{0}}}\right|_{0}^{2}+\left|b^{\alpha_{i_{0}}}-\bar{b}^{\alpha_{i_{0}}}\right|_{0}^{2}\right\}+ \\
+\left\{\left|v_{i_{0}}\right|_{0}\left|\bar{c}^{\alpha_{i_{0}}}-c^{\alpha_{i_{0}}}\right|_{0}+\left|f^{\alpha_{i_{0}}}-\bar{f}^{\alpha_{i_{0}}}\right|_{0}\right\}+ \\
+2 \delta\left\{\ell\left[\bar{\sigma}^{\alpha_{i_{0}}}\right]_{1}^{2}+\left[\bar{b}^{\alpha_{i_{0}}}\right]_{1}+2\right\}\left|x_{0}-y_{0}\right|^{2}+ \\
\left\{\left|\bar{v}_{i_{0}}\right|_{0}\left[\bar{c}^{\alpha_{i_{0}}}\right]_{1}+\left[\bar{f}^{\alpha_{i_{0}}}\right]_{1}+\lambda[\psi]_{1}\right\}\left|x_{0}-y_{0}\right|+C \varepsilon\left(1+\left|x_{0}\right|^{2}+\left|y_{0}\right|^{2}\right) .
\end{gathered}
$$

We set $k_{1}:=\left\{2 \ell\left[\bar{\sigma}^{\alpha_{i_{0}}}\right]_{1}^{2}+2\left[\bar{b}^{\alpha_{i_{0}}}\right]_{1}+4\right\}, k_{2}:=\left\{\left|\bar{v}_{i_{0}}\right|_{0}\left[\bar{c}^{\alpha_{i_{0}}}\right]_{1}+\left[\bar{f}^{\alpha_{i_{0}}}\right]_{1}+\lambda[\psi]_{1}\right\}$. From now on we proceed as in ([1, theorem A.1]). Noting that $2 \phi\left(x_{0}, y_{0}\right) \geq \phi\left(x_{0}, x_{0}\right)+\phi\left(y_{0}, y_{0}\right)$, we have

$$
\left|x_{0}-y_{0}\right| \leq C \delta^{-1}
$$

where $C$ depends $K$. The inequality (73) implies that

$$
\left|x_{0}-y_{0}\right|^{2} \leq C \delta^{-2}
$$

where $C$ depends on $K$. So we obtain

$$
\begin{gathered}
\lambda m \leq\left\{\left|v_{i_{0}}\right|_{0}\left|\bar{c}^{\alpha_{i_{0}}}-c^{\alpha_{i_{0}}}\right|_{0}+\left|f^{\alpha_{i_{0}}}-\bar{f}^{\alpha_{i_{0}}}\right|_{0}\right\}+ \\
+2 \ell \delta\left\{\left|\sigma^{\alpha_{i_{0}}}-\bar{\sigma}^{\alpha_{i_{0}}}\right|_{0}^{2}+\left|b^{\alpha_{i_{0}}}-\bar{b}^{\alpha_{i_{0}}}\right|_{0}^{2}\right\}+C\left(k_{1}+k_{2}\right) \delta^{-1}+C \varepsilon\left(1+\left|x_{0}\right|^{2}+\left|y_{0}\right|^{2}\right) .
\end{gathered}
$$

We know that $v_{i_{0}}(x)-\bar{v}_{i_{0}}(x)-2 \varepsilon|x|^{2} \leq m$, and so

$$
\begin{gathered}
v_{i_{0}}(x)-\bar{v}_{i_{0}}(x) \leq\left\{\left|v_{i_{0}}\right|_{0}\left|\bar{c}^{\alpha_{i_{0}}}-c^{\alpha_{i_{0}}}\right|_{0}+\left|f^{\alpha_{i_{0}}}-\bar{f}^{\alpha_{i_{0}}}\right|_{0}\right\}+ \\
+2 \ell \delta\left\{\left|\sigma^{\alpha_{i_{0}}}-\bar{\sigma}^{\alpha_{i_{0}}}\right|_{0}^{2}+\left|b^{\alpha_{i_{0}}}-\bar{b}^{\alpha_{i_{0}}}\right|_{0}^{2}\right\}+C\left(k_{1}+k_{2}\right) \delta^{-1}+2 \varepsilon|x|^{2}+C \varepsilon\left(1+\left|x_{0}\right|^{2}+\left|y_{0}\right|^{2}\right) .
\end{gathered}
$$

This inequality holds for all $\delta$, and hence we minimize with respect to $\delta$, by noting that for $l>0$,

$$
\min _{\delta>0}\left(l \delta+C \delta^{-1}\right)=C l^{1 / 2} .
$$

Hence, by sending $\varepsilon$ to zero, we obtain

$$
\begin{gathered}
v_{i_{0}}(x)-\bar{v}_{i_{0}}(x) \leq\left\{\left|v_{i_{0}}\right|_{0}\left|\bar{c}^{\alpha_{i_{0}}}-c^{\alpha_{i_{0}}}\right|_{0}+\left|f^{\alpha_{i_{0}}}-\bar{f}^{\alpha_{i_{0}}}\right|_{0}\right\}+ \\
C\left\{\left|\sigma^{\alpha_{i_{0}}}-\bar{\sigma}^{\alpha_{i_{0}}}\right|_{0}^{2}+\left|b^{\alpha_{i_{0}}}-\bar{b}^{\alpha_{i_{0}}}\right|_{0}^{2}\right\}^{1 / 2},
\end{gathered}
$$

where $C$ depends on $K,\left|v_{i_{0}}\right|_{0},\left|\bar{v}_{i_{0}}\right|_{0}$ and $[\psi]_{1}$. Since $\left(s^{2}+t^{2}\right)^{1 / 2} \leq|s|+|t|$, we can conclude.

INRIA 


\section{References}

[1] G. Barles and E.R. Jakobsen. On the convergence rate of approximation schemes for Hamilton-Jacobi-Bellman equations. M2AN. Mathematical Modelling and Numerical Analysis, 36:33-54, 2002.

[2] G. Barles and E.R. Jakobsen. Error bounds for monotone approximation schemes for Hamilton-Jacobi-Bellman equations. To appear, 2003.

[3] G. Barles and P. E. Souganidis. Convergence of approximation schemes for fully nonlinear second order equations. Asymptotic Analysis, 4:271-283, 1991.

[4] J. F. Bonnans, E. Ottenwaelter, and H. Zidani. Numerical schemes for the two dimensional second-order HJB equation. ESAIM: M2AN, 38:723-735, 2004.

[5] J. F. Bonnans and H. Zidani. Consistency of generalized finite difference schemes for the stochastic HJB equation. SIAM J. Numerical Analysis, 41:1008-1021, 2003.

[6] I. Capuzzo-Dolcetta and L.C. Evans. Optimal switching for ordinary differential equations. SIAM J. Control Optim., 22:143-161, 1984.

[7] M.G. Crandall, H. Ishii, and P.-L. Lions. User's guide to viscosity solutions of second order partial differential equations. Bull. American Mathematical Society (New Series), 27:1-67, 1992.

[8] L.C. Evans and A. Friedman. Optimal stochastic switching and the dirichlet problem for the Bellman equation. Trans. Amer. Math. Soc., 253:365-389, 1979.

[9] H. Ishii and S. Koike. Viscosity solutions for monotone systems of second order elliptic PDEs. Comm. Partial Differential Equation, 16:1095-1128, 1991.

[10] H. Ishii and S. Koike. Viscosity solutions of a system of nonlinear second order elliptic PDEs arising in switching games. Funkcial. Ekvac., 34:143-155, 1991.

[11] E. R. Jakobsen. On error bounds for monotone approximation schemes for multidimensional Isaac equations. to appear, 2004.

[12] E.R. Jakobsen and K.H. Karlsen. Continuous dependence estimates for viscosity solutions of fully nonlinear degenerate elliptic equations. Electronic J. Differential Equations, pages 1-10, 2002.

[13] N.V. Krylov. On the rate of convergence of finite difference approximation for Bellman's equation. St. Petersburg Math. J., 9:639-650, 1997.

[14] N.V. Krylov. On the rate of convergence of finite-difference approximations for Bellman's equations with variable coefficients. Probability Theory and Related Fields, 117:1$16,2000$. 
[15] H.J. Kushner and P.G. Dupuis. Numerical methods for stochastic control problems in continuous time, volume 24 of Applications of mathematics. Springer, New York, 2001. Second edition.

[16] N. Yamada. Viscosity solutions for a system of elliptic inequalities with bilateral obstacles. Funkcial. Ekvac., 30:417-425, 1987.

INRIA 


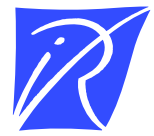

Unité de recherche INRIA Rocquencourt

Domaine de Voluceau - Rocquencourt - BP 105 - 78153 Le Chesnay Cedex (France)

Unité de recherche INRIA Futurs : Parc Club Orsay Université - ZAC des Vignes

4, rue Jacques Monod - 91893 ORSAY Cedex (France)

Unité de recherche INRIA Lorraine : LORIA, Technopôle de Nancy-Brabois - Campus scientifique

615, rue du Jardin Botanique - BP 101 - 54602 Villers-lès-Nancy Cedex (France)

Unité de recherche INRIA Rennes : IRISA, Campus universitaire de Beaulieu - 35042 Rennes Cedex (France)

Unité de recherche INRIA Rhône-Alpes : 655, avenue de l'Europe - 38334 Montbonnot Saint-Ismier (France)

Unité de recherche INRIA Sophia Antipolis : 2004, route des Lucioles - BP 93 - 06902 Sophia Antipolis Cedex (France)

INRIA - Domaine de Voluceau - Rocquencourt, BP 105 - 78153 Le Chesnay Cedex (France)

http://www.inria.fr

ISSN 0249-6399 